Preprint typeset in JHEP style - HYPER VERSION

\title{
Continuity, Deconfinement, and (Super) Yang-Mills Theory
}

\author{
Erich Poppitz, ${ }^{1, *}$ Thomas Schäfer, ${ }^{2, \dagger}$ Mithat Ünsal ${ }^{3, \ddagger}$ \\ ${ }^{1}$ Department of Physics, University of Toronto, Toronto, ON M5S 1A7, Canada \\ ${ }^{2}$ Department of Physics, North Carolina State University, Raleigh, NC 27695, USA \\ ${ }^{3}$ Department of Physics and Astronomy, SFSU, San Francisco, CA 94132, USA
}

AbStract: We study the phase diagram of $S U(2)$ Yang-Mills theory with one adjoint Weyl fermion on $\mathbb{R}^{\mathbf{3}} \times \mathbb{S}^{\mathbf{1}}$ as a function of the fermion mass $m$ and the compactification scale $L$. This theory reduces to thermal pure gauge theory as $m \rightarrow \infty$ and to circle-compactified (nonthermal) supersymmetric gluodynamics in the limit $m \rightarrow 0$. In the $m$ - $L$ plane, there is a line of center-symmetry changing phase transitions. In the limit $m \rightarrow \infty$, this transition takes place at $L_{c}=1 / T_{c}$, where $T_{c}$ is the critical temperature of the deconfinement transition in pure Yang-Mills theory. We show that near $m=0$, the critical compactification scale $L_{c}$ can be computed using semi-classical methods and that the transition is of second order. This suggests that the deconfining phase transition in pure Yang-Mills theory is continuously connected to a transition that can be studied at weak coupling. The center-symmetry changing phase transition arises from the competition of perturbative contributions and monopoleinstantons that destabilize the center, and topological molecules (neutral bions) that stabilize the center. The contribution of molecules can be computed using supersymmetry in the limit $m=0$, and via the Bogomolnyi-Zinn-Justin (BZJ) prescription in non-supersymmetric gauge theory. Finally, we also give a detailed discussion of an issue that has not received proper attention in the context of $N=1$ theories - the non-cancellation of nonzero-mode determinants around supersymmetric BPS and KK monopole-instanton backgrounds on $\mathbb{R}^{3} \times \mathbb{S}^{1}$. We explain why the non-cancellation is required for consistency with holomorphy and supersymmetry and perform an explicit calculation of the one-loop determinant ratio.

\footnotetext{
*poppitz@physics . utoronto.ca

$\dagger$ tmschaef@ncsu.edu

${ }^{\ddagger}$ unsal@sf su. edu
} 


\section{Contents}

$\begin{array}{lr}\text { 1. Introduction } & 1\end{array}$

2. Mass deformation of $\mathcal{N}=1$ super-Yang-Mills on $\mathbb{S}^{1} \times \mathbb{R}^{3} \quad 4$

2.1 Perturbation theory 4

2.2 Non-perturbative effects at $\mathbf{m}=\mathbf{0}$ via supersymmetry 6

2.3 Non-perturbative effects at $\mathbf{m}=\mathbf{0}$ via topological molecules 8

2.3.1 Magnetic bions, quasi-zero modes, and the mass gap for the dual photon 9

2.3.2 Neutral (center-stabilizing) bions and the BZJ prescription 10

2.4 Center-stabilizing vs. center-breaking effects in softly broken SYM 12

3. Pure Yang-Mills theory 14

3.1 Non-perturbative effects on the classical background 15

$\begin{array}{lll}3.1 .1 & \text { Quasi-zero modes and bion amplitudes } & 17\end{array}$

3.1.2 Connecting monopole-instantons to dyon particles 19

4. Comments on the literature $\quad 21$

5. Conclusions and outlook 23

A. Supersymmetry and the non-cancelling nonzero-mode determinants 24

A.1 Calculating the ratio of nonzero mode determinants 26

A.2 Interpretation 30

A.3 Linear-chiral superfield duality 32

B. The monopole-induced potential in softly-broken SYM 35

\section{Introduction}

Consider a quantum mechanical system with a potential with multiple degenerate minima. The ground state energy (as well as the energies of higher eigenstates) has a weak coupling expansion of the form

$$
E(g)=E_{\text {pert. }}+E_{\text {nonpert. }}=E_{0}[1+O(g)]+e^{-1 / g}[1+O(g)]+O\left(e^{-2 / g}\right) .
$$

Since $e^{-1 / g}$ has an essential singularity at $g=0$ it is impossible to express this contribution as a perturbative series in $g$, and hence this term ${ }^{1}$ is intrinsically non-perturbative. Some of the

\footnotetext{
${ }^{1}$ The exponentially small terms may also be multiplied by additional negative powers and logarithms of $g$.
} 
most interesting phenomena in quantum mechanics - tunneling, the absence of spontaneous symmetry breaking, the formation of energy-bands in periodic potentials - are due to $e^{-1 / g}$ effects. Although the leading term and the exponentially small contributions in (1.1) are intertwined in a deep way, as typical inaccuracies of perturbation theory are expressed in terms of functions with essential singularities as above, there is a sense in which (1.1) should be seen as a double expansion, a perturbative expansion in $g$ and a non-perturbative expansion in $e^{-1 / g}$.

In this paper, we will use this double expansion to study the phase diagram of an asymptotically free gauge theory with strong coupling scale $\Lambda$ on $\mathbb{R}^{3} \times \mathbb{S}^{1}$. In a theory without fermions the compactification scale on the $\mathbb{S}^{1}$ circle can always be given a thermal interpretation. At small $\mathbb{S}^{1}$, of size $L \ll \Lambda^{-1}$, it is well-known that such theories are amenable to a perturbative treatment. A less widely appreciated fact is that, if certain conditions are satisfied, such theories are also amenable to non-perturbative semi-classical studies. Let $\Omega=P \exp \left[i \int_{\mathbb{S}^{1}} A_{4} d x_{4}\right]$ denote the gauge holonomy (or Wilson line) in the compact direction, which, classically, is a "flat direction". We expect that quantum effects will induce a potential for the holonomy $\Omega$ of the form:

$$
V(\Omega)=V_{\text {pert. }}(\Omega)+V_{\text {nonpert. }}(\Omega)
$$

where $V_{\text {pert. }}$ is the contribution of the perturbative loop-expansion in $g^{2}$ and $V_{\text {nonpert. }}(\Omega)$ is a non-perturbative expansion, presumably containing terms of the form $e^{-c / g^{2}}$. The perturbative term $V_{\text {pert. }}$ was initially computed in [1], and the calculation was extended to higher order in [2-4]. Although the perturbative potential $V_{\text {pert. }}(\Omega)$ is by now part of the standard books of thermal field theory, $V_{\text {nonpert. }}(\Omega)$ has not received as much attention.

The perturbative calculation of the effective potential for the Wilson line in pure $S U(N)$ Yang-Mills theory on $\mathbb{R}^{3} \times \mathbb{S}^{1}$ with small $L=\beta$ gives [1]:

$$
V_{\text {pert. }}(\Omega)=-\frac{2}{\pi^{2} \beta^{4}} \sum_{n=1}^{\infty} \frac{1}{n^{4}}\left|\operatorname{tr} \Omega^{n}\right|^{2}\left(1+O\left(g^{2}\right)\right),
$$

leading to the conclusion that at small $\beta$ the theory is in a deconfined phase, with broken center-symmetry $\left\langle\frac{1}{N} \operatorname{tr} \Omega\right\rangle=1$. If one thinks in terms of eigenvalues of $\Omega$, the potential (1.3) generates an attraction among the eigenvalues. In other words, the effective mass-squared for the Wilson line is negative.

Based on numerical simulations on the lattice we know that the deconfinement transition in pure Yang-Mills theory takes place at a temperature of order $\Lambda: T_{d}=a \Lambda$ where $a$ is a pure number of order one. At one-loop order in perturbation theory, (1.3) shows that the centersymmetry is broken. Higher order corrections do not alter this conclusion; there is no effect at any order in perturbation theory that competes with center symmetry breaking. Hence, the phase transition must be induced by $V_{\text {nonpert. }}(\Omega)$. Disregarding such non-perturbative effects, one would conclude that one cannot explore the transition as the temperature is lowered, from the deconfined to the confined phase, using weak coupling techniques.

In this work, we propose a strategy to analytically study the center-symmetry changing phase transition in four dimensional gauge theories based on an observation discussed in [5]. 
$\mathrm{SU}(2)$

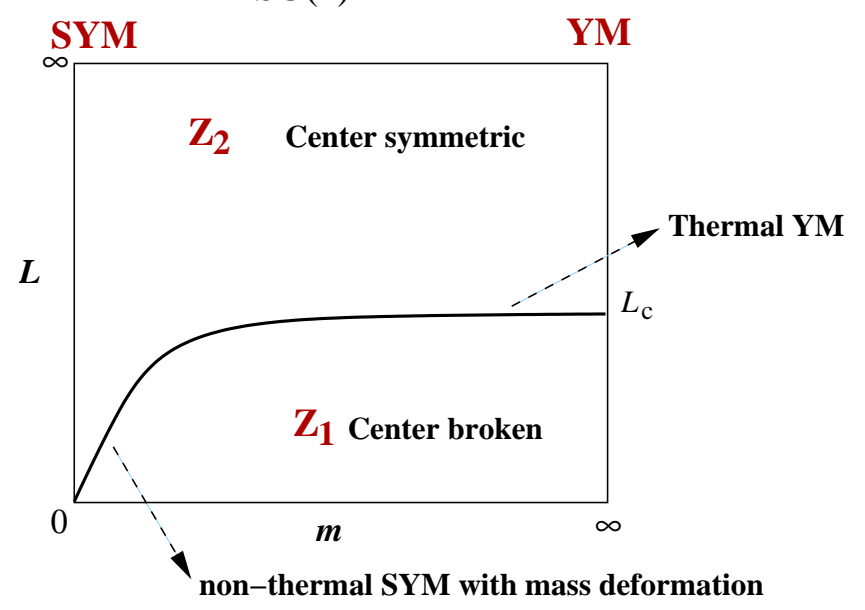

Figure 1: The thermal deconfinement phase transition in pure Yang Mills (YM) theory can be accessed through a non-thermal (quantum) phase transition in supersymmetric Yang Mills (SYM) theory deformed by a gluino mass term. In the massless limit, the supersymmetric theory does not have a phase transition. The phase transition at small- $m$ is analytically calculable and, by decoupling, it is connected to thermal deconfinement phase transition in pure YM theory.

The main idea, schematically shown in Figure 1, is as follows: It is well-known that $\mathcal{N}=1$ SYM with periodic boundary conditions for fermions does not have a phase transition as a function of radius. In fact, for a supersymmetric gauge theory with Hamiltonian $H$ and fermion number operator $F$,

$$
\widetilde{Z}^{\mathrm{SYM}}(L)=\operatorname{tr}\left[e^{-L H}(-1)^{F}\right]
$$

is the supersymmetric (Witten) index and is independent of radius. In softly broken supersymmetric theory, however, this quantity does not have an interpretation as an index. Consider adding a small mass for the fermion in $\mathcal{N}=1$ SYM. Eqn. (1.4) is still well-defined, and can be interpreted as a twisted partition function. The twisted partition function is a signed sum over the states in the bosonic and fermionic Hilbert spaces, $\mathcal{H}_{\mathcal{B}}$ and $\mathcal{H}_{\mathcal{F}}$, according to the $\mathbb{Z}_{2}=(-1)^{F}$ grading,

$$
\widetilde{Z}^{\mathrm{SYM}}(L, m)=Z_{\mathcal{B}}-Z_{\mathcal{F}}=\sum_{n \in \mathcal{H}_{\mathcal{B}}} e^{-L E_{n}}-\sum_{n \in \mathcal{H}_{\mathcal{F}}} e^{-L E_{n}} .
$$

This is different from the ordinary partition function, $Z^{\mathrm{SYM}}(\beta, m)=Z_{\mathcal{B}}+Z_{\mathcal{F}}$ by the over-all sign of the contribution of fermionic states.

The twisted partition function, despite being a non-thermal quantity for general values of the fermion mass $m$, is immensely useful as a tool that continuously connects the thermal phase transition in pure Yang Mills theory with a semi-classically calculable transition on $\mathbb{R}^{3} \times S_{\beta}^{1}$. A similar continuity argument at finite baryon density was made in [6]. For $m \neq 0$, 
(1.5) should be viewed as probing the phase structure of the theory as a function of radius $L$ (which does not generally have an interpretation as inverse temperature). As emphasized, the twisted partition function is manifestly non-thermal. Yet, it can be used to study aspects of a genuine (thermal) deconfinement phase transition in certain limits. This is due to the the following decoupling argument. If the mass of the fermion is infinite, or much larger than the strong scale of $\mathcal{N}=1 \mathrm{SYM}, \widetilde{Z}(L, m)$ reduces to the ordinary thermal partition function of pure Yang-Mills theory:

$$
\left.\widetilde{Z}^{\mathrm{SYM}}(L, m)\right|_{m \rightarrow \infty} \Longrightarrow Z^{\mathrm{YM}}(\beta)=\operatorname{tr}\left[e^{-\beta H}\right], \quad \beta \equiv L
$$

In this limit, because the heavy fermion decouples, we may identify the circumference $L$ with the inverse temperature $\beta$. For a heavy fermion, the choice of the boundary condition is immaterial.

In this work, we will show that the center-symmetry changing phase transition at small $m$ can be computed semi-classically. ${ }^{2}$ In this limit the transition takes place at small $L$, as shown in Figure 1. The physics of the transition is quite interesting. It is based on the competition between topological molecules, called "neutral bions" or "center-stabilizing bions", and semiclassical monopole-instanton effects, as well as perturbative effects. We will argue that these effects are also present at large $m$, in the pure gauge theory, but that in this limit the effect cannot be reliably computed using semi-classical methods.

\section{Mass deformation of $\mathcal{N}=1$ super-Yang-Mills on $\mathbb{S}^{1} \times \mathbb{R}^{3}$}

\subsection{Perturbation theory}

Classical vacua of the theory on $\mathbb{R}^{3} \times \mathbb{S}^{\mathbf{1}}$ are labeled by the expectation value of the Wilson line

$$
\Omega=\exp \left[i \int A_{4} d x_{4}\right] \text {. }
$$

When $L \Lambda \ll 1$, non-zero frequency Kaluza-Klein modes are weakly coupled and may be integrated out perturbatively. If we consider periodic boundary conditions for both the gauge fields and the adjoint Weyl fermions, $A_{\mu}(L)=A_{\mu}(0)$ and $\lambda(L)=+\lambda(0)$, the one-loop effective potential for the Wilson line is $[5,7]$ :

$$
V_{\text {pert. }}^{\mathrm{SYM}}[\Omega, m]=\frac{2}{\pi^{2} L^{4}} \sum_{n=1}^{\infty}\left[-1+\frac{1}{2}(n L m)^{2} K_{2}(n L m)\right] \frac{\left|\operatorname{tr} \Omega^{n}\right|^{2}}{n^{4}} .
$$

\footnotetext{
${ }^{2}$ In order to be precise, we note that the small- $m$, small- $L$ calculability of the transition applies outside of a finite strip around the phase transition line in Figure 1. As usual in second order phase transitions, fluctuations become strong near the critical point and the renormalization group equations describing the critical theory - in the case at hand, in the 3d Ising universality class - are nonperturbative. However, in the weak coupling small- $m$, small- $L$ regime, the width of the strongly fluctuating critical region is small, controlled by powers of the small parameter $L \Lambda$, and the critical values of the mass $m_{c}$ at fixed $L$ (or critical size $L_{c}$ at fixed $m$ ) can be reliably determined up to small corrections.
} 
Here $m$ is the fermion mass and $K_{2}(z)$ is the modified Bessel function of the second kind, with asymptotic behavior

$$
K_{2}(z)= \begin{cases}\frac{2}{z^{2}}-\frac{1}{2}+O\left(z^{2}\right), & z \ll 1 \\ \sqrt{\frac{\pi}{2 z}} e^{-z}, & z \gg 1\end{cases}
$$

As the mass $m \rightarrow \infty$, the fermions decouple regardless of their boundary conditions, and the effective potential (2.2) reduces to the pure gauge result given in (1.3), with the identification $L=\beta$ :

$$
\left.V_{\text {pert. }}^{\mathrm{SYM}}[\Omega, m]\right|_{m \rightarrow \infty}=V_{\text {pert. }}^{\mathrm{YM}}[\Omega]\left(1+O\left(e^{-L m}\right)\right) .
$$

In the opposite limit of massless fermion, the one-loop potential vanishes:

$$
V_{\text {pert. }}^{\mathrm{SYM}}[\Omega, m=0]=0 \text {. }
$$

In fact, because of supersymmetry and the fact that (perturbatively) the theory possesses a moduli space of vacua, the $m=0$ SYM theory does not generate a potential for the Wilson line to any order in perturbation theory. At a typical point on the moduli space, a nonzero background Wilson line is turned on:

$$
\Omega=\left(\begin{array}{cc}
e^{i \Delta \theta / 2} & \\
& e^{-i \Delta \theta / 2}
\end{array}\right)
$$

and the $S U(2)$ gauge group abelianizes down to $U(1)$. Here, $\Delta \theta$ is the separation between the eigenvalues of the Wilson line. Non-perturbatively, the moduli space is lifted due to effects leading to $\Delta \theta=\pi$, i.e., to a center-symmetric holonomy. This effect will be described in two complementary ways in the next Section.

We now turn on a small mass corresponding to soft supersymmetry breaking, $m \ll \Lambda$. In the small $L \Lambda \lesssim 1$ regime, we also have $m L \ll 1$. In this case, using the small- $z$ asymptote of (2.3), we observe that the leading term at $O\left(\mathrm{~m}^{0}\right)$ cancels and an $O\left(\mathrm{~m}^{2}\right)$ potential is induced. The effective potential becomes (up to $O\left(m^{4}\right)$ corrections):

$$
V_{\mathrm{SYM}}[\Omega]=-\frac{m^{2}}{2 \pi^{2} L^{2}} \sum_{n=1}^{\infty} \frac{1}{n^{2}}\left|\operatorname{tr} \Omega^{n}\right|^{2}=-\frac{m^{2}}{L^{2}} B_{2}\left(\frac{\Delta \theta}{2 \pi}\right),
$$

where $B_{2}(x)=x^{2}-x+\frac{1}{6}$ is the second Bernoulli polynomial (the last equality above is valid when $\Delta \theta \in[0,2 \pi])$.

Within the domain of validity of the perturbative analysis, (2.7) shows that Wilson lines with all winding numbers are unstable when the fermion mass is non-zero, despite the use of periodic boundary condition for fermions. Consequently, the $n_{\mathrm{f}}=1$ theory at any non-zero mass $m$ and sufficiently small $L$ will have completely broken center symmetry. On the other hand, the fact that the one loop potential is small, of $O\left(\mathrm{~m}^{2}\right)$, implies that exponentially small semi-classical effects can compete with the perturbative potential. 


\subsection{Non-perturbative effects at $\mathbf{m}=0$ via supersymmetry}

In the classical background (2.6), and at weak coupling, the Wilson line (2.1) behaves as an adjoint Higgs field. The theory at short distances is described by non-abelian $S U(2)$, and at long distances, it is described by an abelian $U(1)$ subgroup. Using abelian duality, $\epsilon_{\mu \nu \lambda} \partial_{\lambda} \sigma=\frac{4 \pi L}{g^{2}} F_{\mu \nu}$ for $\mu, \nu=1,2,3$, we may map the gauge field to a spin-zero dual-photon $\sigma$. It is also useful to define the exponent of gauge holonomy (2.6):

$$
b \equiv \frac{4 \pi}{g^{2}} \Delta \theta .
$$

The kinetic terms of the fields $\sigma$ and $b$ are

$$
\mathcal{L}=\frac{1}{2} \frac{g^{2}}{(4 \pi)^{2} L}\left[\left(\partial_{i} b\right)^{2}+\left(\partial_{i} \sigma\right)^{2}\right] .
$$

In terms of superfields this corresponds to a Kähler potential:

$$
K=\frac{g^{2}}{2(4 \pi)^{2} L} \mathbf{B}^{\dagger} \mathbf{B}
$$

for the chiral superfield $\mathbf{B}$, whose lowest component ${ }^{3}$ is $b-i \sigma$; the fermionic component is the component of the gluino field $\lambda$ which remains massless along the Coulomb branch (2.6). The effective Lagrangian following from (2.10) gives the long-distance perturbative description of the theory on $\mathbb{R}^{3} \times \mathbb{S}^{1}$ - essentially a massless free-field theory.

The non-perturbative dynamics of the theory is quite rich: due to the compact topology of the "adjoint Higgs" (2.1), there are two types of elementary monopole-instantons, $\mathcal{M}_{1}$ and $\mathcal{M}_{2}$. These are sometimes called $3 \mathrm{~d}$ instanton and twisted-instanton, or BPS-monopoleinstanton and KK-monopole-instanton, see $[8,9]$. The Nye-Singer index theorem implies two fermionic zero-modes for each $[10,11]$. The $4 \mathrm{~d}$ BPST instanton (in the long distance regime) can be viewed as a composite of these two. The monopole-instantons give rise to 't Hooft vertices, or amplitudes, of the form:

$$
\begin{aligned}
\mathcal{M}_{1} & =e^{-b+i \sigma} \lambda \lambda, & \mathcal{M}_{2} & =\eta e^{+b-i \sigma} \lambda \lambda, \\
\overline{\mathcal{M}}_{1} & =e^{-b-i \sigma} \bar{\lambda} \bar{\lambda}, & \overline{\mathcal{M}}_{2} & =\eta e^{+b+i \sigma} \bar{\lambda} \bar{\lambda},
\end{aligned}
$$

where $\eta=e^{-\frac{8 \pi^{2}}{g^{2}}}=e^{-2 S_{0}}$ is the $4 \mathrm{~d}$ instanton amplitude (we set the topological theta angle to zero). Since (2.11) carry just two zero modes, they generate a superpotential, given by:

$$
W_{\mathbb{R}^{3} \times \mathbb{S}^{1}}=\frac{M_{P V}^{3} L}{g^{2}}\left(e^{-\mathbf{B}}+\eta e^{\mathbf{B}}\right)=\frac{2 M_{P V}^{3} L}{g^{2}} e^{-S_{0}} \cosh \left(\mathbf{B}-\frac{4 \pi^{2}}{g^{2}}\right)
$$

\footnotetext{
${ }^{3}$ We note that the relation between $\mathbf{B}$ and $b$ is, in fact, nonlinear, see eqn. (A.35), due to the perturbative corrections to the moduli space metric along the Coulomb branch. These are also reflected in the noncancellation of the one-loop fermion and boson nonzero mode determinants around the BPS and KK monopoleinstantons, see Appendix A for a detailed discussion. As these subtleties represent subleading corrections to the Kähler metric (2.10), we ignore them in the main text.
} 
where the coupling is normalized at the cutoff scale $M_{P V}$; details of the instanton calculation leading to (2.12) can be found in [12]. Thus, the infrared Lagrangian is given by (2.10) and (2.12):

$$
\mathcal{L}=\int d^{4} \theta K+\left(\int d^{2} \theta W+\text { h.c. }\right)
$$

and the scalar potential can be easily found:

$$
V(b, \sigma) \equiv K_{\mathbf{B}^{\dagger} \mathbf{B}}^{-1}\left|\frac{\partial W}{\partial \mathbf{B}}\right|^{2}=\frac{64 \pi^{2} M_{P V}^{6} L^{3} e^{-2 S_{0}}}{g^{6}}\left(\cosh \left(2\left(b-\frac{4 \pi^{2}}{g^{2}}\right)\right)-\cos 2 \sigma\right),
$$

where $K_{\mathbf{B}^{\dagger} \mathbf{B}}$ is the mixed second derivative of the Kähler potential. Furthermore, it is convenient to introduce, instead of $b$ of eqn. (2.8), the shifted field:

$$
b \equiv \frac{4 \pi^{2}}{g^{2}}+b^{\prime}, \quad \frac{\Delta \theta}{2 \pi}=\frac{g^{2}}{8 \pi^{2}} b^{\prime}+\frac{1}{2} .
$$

Finally, to rewrite the potential in terms of the strong-coupling scale $\Lambda$ and the $\mathbb{S}^{1}$ size $L$, we use the relations:

$$
\frac{M_{P V}^{3}}{g^{2}} e^{-\frac{4 \pi^{2}}{g^{2}}}=\Lambda^{3}, \quad \frac{4 \pi^{2}}{g^{2}} \approx 3 \log \frac{1}{\Lambda L} .
$$

These relations express the fact that the scale for the coupling of the effective theory is set by the compactification scale $L$. We use the one-loop running coupling constant in the prefactors and two-loop running in the exponent of the instanton amplitude.

Thus, using (2.15) and (2.16), we obtain the final expression for the scalar potential (2.14):

$$
V_{\text {bion }}\left(b^{\prime}, \sigma\right)=48 L^{3} \Lambda^{6} \log \frac{1}{\Lambda L}\left(\cosh 2 b^{\prime}-\cos 2 \sigma\right) .
$$

Note that if the superpotential $W$ is determined by BPS and KK monopole-instantons then the scalar potential is governed by (correlated) monopole-anti-monopole pairs. We have therefore denoted the scalar potential by $V_{\text {bion }}$. We will make this relationship more explicit in the following Section.

The potential $V_{\text {bion }}$ for the $b^{\prime}$-field, $\sim \cosh \left(2 b^{\prime}\right)$, generates a non-perturbative repulsive interaction between the eigenvalues of the Wilson line around $\mathbb{S}^{1}$ : it is minimized at $\left\langle b^{\prime}\right\rangle=0$, which, from (2.15), corresponds to maximally separated eigenvalues, $\langle\Delta \theta\rangle=\pi$, or in terms of the Wilson line:

$$
\langle\Omega\rangle=\left\langle e^{i A_{4} L}\right\rangle=\left(\begin{array}{rl}
e^{i \frac{\pi}{2}} & \\
& e^{-i \frac{\pi}{2}}
\end{array}\right), \quad\langle\operatorname{tr} \Omega\rangle=0,
$$

up to gauge rotations. This is the center-symmetric vacuum of the theory on $\mathbb{R}^{3} \times \mathbb{S}^{1}$. On the other hand, the $V_{\text {bion }}$ potential for the $\sigma$ field has two minima, located at

$$
\langle\sigma\rangle=\{0, \pi\},
$$

associated with discrete chiral symmetry breaking. Evidently, in the effective lagrangian, the mass gap for gauge fluctuations $\sigma$ is generated by the operator $e^{ \pm 2 i \sigma}$, and for the spin-zero scalar (the fluctuation of gauge holonomy $b^{\prime}$ ) it is generated by $e^{ \pm 2 b^{\prime}}$. 
Expanding the action around the center-symmetric gauge holonomy and using component notation, we find the effective lagrangian:

$$
\begin{aligned}
\mathcal{L}= & \frac{1}{2} \frac{g(L)^{2}}{(4 \pi)^{2} L}\left[\left(\partial_{i} b^{\prime}\right)^{2}+\left(\partial_{i} \sigma\right)^{2}\right]+i \frac{L}{g^{2}} \bar{\lambda} \sigma_{i} \partial_{i} \lambda+\alpha e^{-\frac{4 \pi^{2}}{g^{2}(L)}}\left[\left(e^{-b^{\prime}+i \sigma}+e^{+b^{\prime}-i \sigma}\right) \lambda \lambda+\text { c.c. }\right] \\
& +\beta \frac{e^{-\frac{8 \pi^{2}}{g^{2}(L)}}}{L^{3}}\left[e^{-2 b^{\prime}}+e^{2 b^{\prime}}-e^{-2 i \sigma}-e^{2 i \sigma}\right] .
\end{aligned}
$$

We expressed the Lagrangian in component notation to elucidate the physical origin of the various terms ${ }^{4}$.

\subsection{Non-perturbative effects at $\mathrm{m}=0$ via topological molecules}

We now provide a derivation of the bosonic potential without the use of supersymmetry. This formalism will apply to both supersymmetric and non-supersymmetric theories. As discussed in Refs. $[13,14]$, in general gauge theories with massless adjoint fermions (i.e., not only in the supersymmetric single massless Weyl flavor case $n_{\mathrm{f}}=1$ ), the $3 \mathrm{~d}$ instanton and twisted instanton do not generate a mass gap for the gauge fluctuations because of their fermionic zero mode structure. The zero modes are determined by the index theorem [10,11], and the corresponding instanton amplitudes have the form given in (2.11) or the generalization thereof for the $n_{\mathrm{f}}>1$ case.

Unlike the superpotential, which arises due to monopole-instantons with exactly two zero modes, the associated bosonic potential must be induced by topological molecules which do not have any fermionic zero modes. The bosonic potential is generated by correlated monopole-anti-monopole pairs. To second order in the semi-classical expansion, the possibilities are the following: $\left[\mathcal{M}_{1} \overline{\mathcal{M}}_{1}\right],\left[\mathcal{M}_{2} \overline{\mathcal{M}}_{2}\right],\left[\mathcal{M}_{1} \overline{\mathcal{M}}_{2}\right],\left[\mathcal{M}_{2} \overline{\mathcal{M}}_{1}\right]$. These objects can be viewed as composites of (2.11). The magnetic and topological charges and the amplitudes associated with these instanton-anti-instanton events are:

$\begin{array}{ccc}\text { composite } & \left(Q_{m}, Q_{T}\right) & \text { amplitude } \\ {\left[\mathcal{M}_{1} \overline{\mathcal{M}}_{1}\right]} & (0,0) & e^{-2 b^{\prime}} \\ {\left[\mathcal{M}_{2} \overline{\mathcal{M}}_{2}\right]} & (0,0) & e^{+2 b^{\prime}} \\ {\left[\mathcal{M}_{1} \overline{\mathcal{M}}_{2}\right]} & (+2,0) & e^{+2 i \sigma} \\ {\left[\mathcal{M}_{2} \overline{\mathcal{M}}_{1}\right]} & (-2,0) & e^{-2 i \sigma}\end{array}$

The action and interaction due to massless boson exchange of two fundamental monopole instantons with (bosonic) amplitudes $e^{n_{b} b^{\prime}+i n_{m} \sigma}$ and $e^{n_{b}^{\prime} b^{\prime}+i n_{m}^{\prime} \sigma}$ is (here $n_{b}, n_{m}= \pm 1$ ):

$$
S(r)=2 S_{0}+S_{\mathrm{int}}=\frac{8 \pi^{2}}{g^{2}}+\left(-n_{b} n_{b}^{\prime}+n_{m} n_{m}^{\prime}\right) \frac{4 \pi L}{g^{2} r}
$$

\footnotetext{
${ }^{4}$ In order not to clutter notation, we kept only the exponential dependence of the coupling $g^{2}(L)$ in the non-perturbative terms in $(2.20)$, i.e., we absorbed the numerical coefficients and the $\log \frac{1}{L \Lambda}$ dependence in the prefactors $\alpha$ and $\beta$; these can be recovered from (2.12), (2.17).
} 
where $r=|\mathbf{r}|$ is the separation between two instanton events. The interaction proportional to $n_{b} n_{b}^{\prime}$ is due to the exchange of the massless $A_{4}$ modulus and the one proportional to $n_{m} n_{m}^{\prime}$ is due to the exchange of the dual photon ("magneto static"). The interaction term is repulsive for $\left(-n_{b} n_{b}^{\prime}+n_{m} n_{m}^{\prime}\right)>0$, attractive for $\left(-n_{b} n_{b}^{\prime}+n_{m} n_{m}^{\prime}\right)<0$ and zero otherwise. The fermion-induced interactions will be considered below.

\subsubsection{Magnetic bions, quasi-zero modes, and the mass gap for the dual photon}

All the topological molecules contributing to the bosonic potential have vanishing topological charge, i.e., they are indistinguishable from the perturbative vacuum in that sense. However, the $\left[\mathcal{M}_{1} \overline{\mathcal{M}}_{2}\right]$ (and its anti-molecule) events carry two units of magnetic charge. The prefactor of the amplitude can be found as follows.

The $\mathcal{M}_{1}$ instanton has four bosonic zero modes. Three of these are the positions $\mathbf{x} \in \mathbb{R}^{3}$ and one is related to the internal $U(1)$ symmetry. Note that there is no size modulus associated with monopole-instantons, unlike the $4 \mathrm{~d}$ BPST instanton. This is one of the reasons that we can do reliable semi-classical analysis. Let an $\overline{\mathcal{M}}_{2}$ be located at $\mathbf{y}$. Because of the interaction which depends on the separation of the two events, $\mathbf{r}=\mathbf{x}-\mathbf{y}$, the relative coordinate is no longer an exact zero mode, while the "center of mass" position $\mathbf{R}=(\mathbf{x}+\mathbf{y}) / 2$ of the two events is an exact zero mode. The relative coordinate is now a quasi-zero mode. This is to say that the operator of the quadratic fluctuations in the background of $\mathcal{M}_{1}$ and $\overline{\mathcal{M}}_{2}$ has, in its spectrum, an exact zero mode, a low lying quasi-zero mode and parametrically separated Gaussian fluctuations. The latter modes can be trivially integrated out. The zero and quasizero modes are particularly important. In particular, the integrals over the quasi-zero modes need to be done exactly.

The magnetic bion amplitude associated with a bion located at $\mathbf{R}$ can be found by integrating over the quasi-zero mode exactly. Below, we write the expression for the $n_{\mathrm{f}}$ flavor theory for later convenience. The amplitude associated with an $\left[\mathcal{M}_{1} \overline{\mathcal{M}}_{2}\right]$ composite is:

$$
\left[\mathcal{M}_{1} \overline{\mathcal{M}}_{2}\right] \sim \mathcal{A} e^{-2 S_{0}} e^{2 i \sigma}
$$

where

$$
\mathcal{A}=\int d^{3} r e^{-\left(2 \times \frac{4 \pi L}{g^{2} r}+4 n_{f} \log r\right)}=4 \pi I\left(\lambda, n_{f}\right), \quad \lambda \equiv \frac{g^{2}}{8 \pi L}
$$

The meaning of the terms in the exponent is as follows: $2 \times \frac{4 \pi L}{g^{2} r}$ accounts for the repulsion due to exchange of $\sigma$ and $b$-scalars, and $4 n_{f} \log r$ is the attraction due to fermion zero mode exchange. Consequently, there is a single saddle-point in the quasi-zero mode integral, given (for $n_{\mathrm{f}}=1$ ) by:

$$
r_{\mathrm{b}}=\frac{4 \pi L}{g^{2}}
$$

which can be interpreted as the magnetic bion size. The bion size is much larger than monopole-size, but much smaller than (uncorrelated) inter-monopole separation. Consequently, a representation of the partition function as a dilute gas of magnetic bions is justified. 
The integral in (2.24) is given by:

$$
I\left(\lambda, n_{f}\right)=\int d r e^{-\left(\frac{1}{\lambda r}+\left(4 n_{f}-2\right) \log r\right)}=\lambda^{4 n_{f}-3} \Gamma\left(4 n_{f}-3\right),
$$

where $n_{\mathrm{f}}=1$ for SYM. The way to check that this is self-consistent is as follows. The interaction term for the magnetic bion in (2.22) must be parametrically smaller than the leading action in order for the relative coordinate to deserve the name quasi-zero mode. Just saying that $2 S_{0} \gg S_{\text {int }}$, or equivalently, $r \gg \frac{L}{\pi}$, is not sufficiently good, because this does not preclude the $O(1)$ changes in the combined action, $2 S_{0}$. It must be such that $\left|S_{\text {int }}\right| \sim O\left(g^{2}\right) \times\left(2 S_{0}\right) \ll\left(2 S_{0}\right)$. Indeed, the action of the magnetic bion configuration associated with $r=r_{\mathrm{b}}$, given in $(2.22)$, takes the form

$$
S\left(r_{\mathrm{b}}\right)=2 S_{0}\left(1+O\left(g^{2}\right)\right)
$$

Since the interaction changes the action only by parametrically small $O\left(g^{2}\right)$ effects, the magnetic bion topological molecule can be viewed as a quasi-solution.

The magnetic bion molecules described in this Section are responsible for the generation of a mass gap for the dual photon (the potential for $\sigma$ in $(2.20)$ ) in SYM on $\mathbb{R}^{3} \times \mathbb{S}^{1}$, and they generate the confining string tension. In the following Section we will study a second type

of topological molecule, which is more subtle to identify, but plays an important role in the center-symmetry realization.

\subsubsection{Neutral (center-stabilizing) bions and the BZJ prescription}

Consider now the other possible composite from the list (2.21), the $\left[\mathcal{M}_{1} \overline{\mathcal{M}}_{1}\right]$ composite which carries no magnetic and topological charge (the $\left[\mathcal{M}_{2} \overline{\mathcal{M}}_{2}\right]$ is treated similarly). Here, the integral over the quasi-zero mode is, naively:

$$
\left[\mathcal{M}_{1} \overline{\mathcal{M}}_{1}\right] \sim \mathcal{A}_{\text {naive }} e^{-2 S_{0}} e^{ \pm 2 b^{\prime}}
$$

where:

$$
\begin{aligned}
\mathcal{A}_{\text {naive }}\left(g^{2}\right) & =\int d^{3} r e^{-\left(-2 \times \frac{4 \pi}{g^{2} r}+4 n_{f} \log r\right)}=4 \pi \tilde{I}\left(\lambda, n_{f}\right), \\
\tilde{I}\left(\lambda, n_{f}\right) & =\int d r e^{-\left(-\frac{1}{\lambda r}+\left(4 n_{f}-2\right) \log r\right)} .
\end{aligned}
$$

Now, the interactions between constituents due to $\sigma$ and $b$ exchange are both attractive, while the fermion zero mode induced attraction is not altered (it remains attractive). The integral, (2.29), as it stands, is dominated by the small $r$ regime, where not only (2.29) is incorrect, it is also hard to make sense of constituents as the interaction becomes large. This is in sharp contrast with the magnetic bion (2.26) $[14,15]$.

At first sight, this may seem to prevent us from computing the contribution from these pairs, but this is not actually the case. We will reach a satisfactory resolution of the problem, via the Bogomolnyi-Zinn-Justin prescription: The integrals over the quasi-zero modes of attractive instanton-anti-instanton molecules can be calculated in a manner initially described 
by Bogomolnyi [16] in the context of quantum mechanics. The relation between this prescription and the Borel procedure was pointed out in the same context by Zinn-Justin [17], see also [18]. The prescription is to analytically continue the coupling $g^{2}$ in the instantonanti-instanton interaction to $-g^{2}$. This turns the attractive Coulomb force into a repulsive one. Then we calculate the resulting integral exactly, without any Gaussian approximations. Finally, we analytically continue the final result back to positive $g^{2}$.

Recall that, very often for a non-Borel summable series, when $g^{2}$ is continued to $-g^{2}$, the series become Borel summable. However, one needs to continue back to positive $g^{2}$. Depending on the path that one takes the coupling to the positive $g^{2}$, the Borel sum typically produces an ambiguous (non-perturbative) imaginary part. This is a manifestation of non-Borel summability. In the quantum mechanical examples that Refs. [16] and [17] studied, the ambiguity in the Borel sum is canceled by the ambiguity associated with the attractive instanton-anti-instanton molecule. The prescription for the topological molecules may be viewed as consistently extending the Borel prescription for perturbative sums to non-perturbative sectors with vanishing quantum numbers. For a fuller discussion of these phenomena in field theory, see [19].

This prescription will give an overall phase between the magnetic bion amplitude and center-stabilizing bion amplitude. This phase difference is physical and crucial for our considerations. Following the prescription, when we modify $\tilde{I}\left(\lambda, n_{f}\right) \rightarrow \tilde{I}\left(-\lambda, n_{f}\right)$ (recall that $\left.\lambda \equiv g^{2} / 8 \pi L\right)$ the Coulomb-interaction becomes repulsive and we can evaluate the integral over the quasi-zero mode. In fact, it is equal to the integral $I\left(\lambda, n_{f}\right)$ for the magnetic bion computed in the previous Section. Next, we substitute $g^{2} \rightarrow-g^{2}(\lambda \rightarrow-\lambda)$ to obtain the final result for the center-stabilizing bion amplitude. To summarize, the generalization of the BZJ prescription to field theory results in the chain:

$$
\tilde{I}\left(\lambda, n_{f}\right) \rightarrow \tilde{I}\left(-\lambda, n_{f}\right)=I\left(\lambda, n_{f}\right) \rightarrow I\left(-\lambda, n_{f}\right)=(-\lambda)^{3-4 n_{f}} \Gamma\left(4 n_{f}-3\right)=-I\left(\lambda, n_{f}\right) .
$$

The last equality is only valid for integer $n_{f}$ and gives an overall sign of the center-stabilizing bion amplitude opposite that for the magnetic bion. We note that this line of reasoning has a close parallel in supersymmetric quantum mechanics [21].

The importance of the relative sign between the magnetic bion amplitude and centerstabilizing bion amplitude is worth noting, as it is a physical consequence of our prescription. As a result, we obtain for their combined contribution:

$$
\begin{aligned}
V(b, \sigma) & \sim \eta \cosh 2 b^{\prime}-\eta \cos 2 \sigma=e^{-2 S_{0}}\left[\left(1+2 b^{\prime 2}+\ldots\right)-\left(1-2 \sigma^{2}+\ldots\right)\right] \\
& =2 e^{-2 S_{0}}\left(b^{\prime 2}+\sigma^{2}\right)
\end{aligned}
$$

the same result that we obtained earlier by using holomorphy. The crucial point here is the cancellation of the "cosmological constant" term in the potential. Recall that in a supersymmetric theory with unbroken supersymmetry, the expectation value of Hamiltonian is positive semi-definite and $\left\langle\Psi_{n}|H| \Psi_{n}\right\rangle \geq 0$, and that the bound is saturated for the ground state $\left\langle\Psi_{0}|H| \Psi_{0}\right\rangle=0$. If the relative sign was not present, the ground state energy would not 
vanish, implying a breakdown of supersymmetry. Equally importantly, the absence of the relative sign between the $\left[\mathcal{M}_{1} \overline{\mathcal{M}}_{1}\right]$ and $\left[\mathcal{M}_{1} \overline{\mathcal{M}}_{2}\right]$ amplitudes would lead to the presence of a relative sign between the mass term for the two scalars of the form $\left(b^{2}-\sigma^{2}\right)$, signaling an instability. Clearly, neither is the case.

\subsection{Center-stabilizing vs. center-breaking effects in softly broken SYM}

As explained in Section 2.1 there is no perturbative contribution to the Wilson line effective potential for $m=0$, but there is a non-perturbatively induced potential which ensures unbroken center symmetry in the supersymmetric theory on $\mathbb{R}^{3} \times S^{1}$. This potential, as explained above, is due to center-stabilizing bions.

We now turn on a small but non-zero $m$. At small $L$, one expects a competition between the one-loop $O\left(\mathrm{~m}^{2}\right)$ potential for the Wilson line and the non-perturbatively induced superpotential, leading to non-uniformity in the $m \rightarrow 0$ and $L \rightarrow 0$ limits. Taking $m \rightarrow 0$ first, the theory lands on the center-symmetric phase. If, instead, the $L \rightarrow 0$ limit is taken first, the theory lands on the center-broken phase. The transition line separating center-symmetric and center-broken phases must emerge from the $L=m=0$ corner of the phase diagram, as illustrated for an $S U(2)$ theory on Fig. 1. Let us now describe the center-symmetry breaking dynamics in some more detail.

Adding a soft mass term for the fermions reduces the $\mathcal{N}=1$ supersymmetry to $\mathcal{N}=0$ and has the effect of lifting the fermion zero modes from the instanton amplitudes (2.11). The mass perturbation is:

$$
\Delta \mathcal{L}_{m}=\frac{m}{g^{2}} \operatorname{tr} \lambda \lambda+\text { h.c. . }
$$

The insertion of mass terms lifts the zero modes of the monopole-instanton amplitudes (2.11) which now contribute to the potential for $\sigma$ and $b$. The corresponding calculation is presented in the appendix, and the result for the monopole-instanton contribution to the scalar potential, to leading order in $m(m L \ll 1)$, takes the form:

$$
V_{\text {mon. }}=24 m L \Lambda^{3} \cos \sigma\left(\log \frac{1}{\Lambda L} \cosh b^{\prime}-\frac{1}{3} b^{\prime} \sinh b^{\prime}\right) .
$$

Despite the addition of a fermion mass term, the fermion-attraction mechanism giving rise to magnetic and center-stabilizing bions is still operative, provided the fermion mass is smaller

than the inverse size of the bions, $r_{\mathrm{b}}=\frac{4 \pi L}{g^{2}}$ from (2.25), i.e., for $m L<\frac{g^{2}}{4 \pi}$ (below, we shall see that this condition is obeyed in the regime where we can study the competition between center-breaking and center-stabilizing effects). Thus, adding the bion and monopole non-perturbative contributions (eqns. (2.17) and (2.33), respectively) to the the perturbative contribution (2.7), recalling (2.15), we obtain the full scalar potential:

$$
\begin{aligned}
V_{\text {total }}= & 48 L^{3} \Lambda^{6} \log \frac{1}{\Lambda L}\left(\cosh 2 b^{\prime}-\cos 2 \sigma\right) \\
& +24 m L \Lambda^{3} \cos \sigma\left(\log \frac{1}{\Lambda L} \cosh b^{\prime}-\frac{1}{3} b^{\prime} \sinh b^{\prime}\right)-\frac{m^{2}}{36 L \log ^{2} \frac{1}{\Lambda L}}\left(b^{\prime}\right)^{2} .
\end{aligned}
$$


It is convenient to introduce dimensionless masses, compactification scale, and potential:

$$
\tilde{m} \equiv \frac{m}{\Lambda}, \quad \tilde{L} \equiv \Lambda L, \quad \tilde{V} \equiv \frac{L^{3} V_{\text {total }}}{48 \tilde{L}^{6} \log \tilde{L}^{-1}} .
$$

The final result for the scalar potential of the mass-deformed SYM theory is:

$$
\begin{aligned}
\tilde{V}= & \cosh 2 b^{\prime}-\cos 2 \sigma+\frac{1}{2} \frac{\tilde{m}}{\tilde{L}^{2}} \cos \sigma\left(\cosh b^{\prime}-\frac{1}{3 \log \tilde{L}^{-1}} b^{\prime} \sinh b^{\prime}\right) \\
& -\frac{1}{1728}\left(\frac{\tilde{m}}{\tilde{L}^{2}}\right)^{2} \frac{1}{\log ^{3} \tilde{L}^{-1}}\left(b^{\prime}\right)^{2} .
\end{aligned}
$$

The physics that this potential encapsulates is our main result. Before we study the relative importance of the various terms in (2.36), let us summarize the region of validity of the scalar potential. It was derived using weak-coupling semi-classical calculations at small $L$, whose validity requires that $\Lambda L \ll 1$. The validity of the fermion-pairing bion mechanism further requires $m L \log \frac{1}{\Lambda L} \ll 1$; the usual soft-breaking condition $m \ll \Lambda$ is then automatically satisfied. In other words, both dimensionless parameters $\tilde{m}$ and $\tilde{L}$ from (2.35) are small.

We can now use the potential (2.36) to study the symmetry realization of the theory as the parameters are varied:

1. Consider the domain:

$$
\frac{\tilde{m}}{8 \tilde{L}^{2}} \ll 1,
$$

In this domain, the bion-induced center-stabilizing term $\left(\cosh 2 b^{\prime}\right)$ dominates over both the monopole and perturbative contributions, both of which favor center-symmetry breaking, as we show below. In the regime of small $\frac{\tilde{m}}{8 \tilde{L}^{2}}$, with $\tilde{m}>0$ (recall that we set the $\theta$-angle to zero), the vacuum with $\langle\sigma\rangle=\pi,\left\langle b^{\prime}\right\rangle=0$ represents the global minimum of $\tilde{V}$, while the one with $\langle\sigma\rangle=0$ is only metastable. In this regime, $\langle\operatorname{tr} \Omega\rangle=0$, with unbroken center symmetry. To see this explicitly, we expand (2.36) around $\langle\sigma\rangle=\pi$, $\left\langle b^{\prime}\right\rangle=0$, to quadratic order in the fluctuations $\delta \sigma$ and $\delta b^{\prime}$ :

$$
\begin{aligned}
\tilde{V}= & 2\left(1-\frac{\tilde{m}}{8 \tilde{L}^{2}}\left[1+\frac{2}{3 \log \tilde{L}}-\frac{\tilde{m}}{\tilde{L}^{2}} \frac{1}{432 \log ^{3} \tilde{L}}\right]\right)\left(\delta b^{\prime}\right)^{2} \\
& +2\left(1+\frac{\tilde{m}}{8 \tilde{L}^{2}}\right)(\delta \sigma)^{2}
\end{aligned}
$$

where we have dropped the constant $\tilde{V}\left(b^{\prime}=0, \sigma=\pi\right)$. In the regime (2.37) there is a mass gap for the dual photon and the theory is in the confining phase with unbroken center-symmetry.

2. It is clear from (2.38) that, as we depart the regime (2.37), center-symmetry becomes destabilized. The leading center-breaking effect, in the region where the semi-classical analysis is valid, is due to the non-perturbative monopole-instanton term, which tends 
to reduce the mass of the Wilson line. The effect of the center-destabilizing perturbative contribution, given by the last term in (2.36), is suppressed both numerically and parametrically, by the large factor $\left|\log ^{3} \tilde{L}\right|$.

Thus, keeping $\tilde{m}$ small and fixed, as we further decrease $\tilde{L}$, the theory leaves the confining domain (2.37). The monopole term becomes the most dominant and destabilizes the $\left\langle b^{\prime}\right\rangle=0$ center-symmetric vacuum. As eqn. (2.38) shows, the center-symmetry destabilization is continuous. At $\tilde{L}=\tilde{L}_{c}$ where

$$
\tilde{L}_{c}^{2}=\frac{\tilde{m}}{8}\left[1+\mathcal{O}\left(\frac{1}{\log \tilde{L}}, \frac{\tilde{m}}{\tilde{L}^{2} \log ^{3} \tilde{L}}\right)\right]
$$

the center-symmetric vacuum gets destabilized and the two eigenvalues of the holonomy smoothly approach each other.

The potential (2.36) can be used to study the physics until the semi-classical description breaks down (this occurs when the scale of $S U(2) \rightarrow U(1)$ breaking governed by the eigenvalue difference $\Delta \theta$ times $\frac{1}{L}$ becomes comparable to the strong-coupling scale $\Lambda$ ). The evolution of the eigenvalues leads to spontaneous breaking of the $\mathbb{Z}_{2}$ center symmetry, $\left\langle\frac{1}{2} \operatorname{tr} \Omega\right\rangle= \pm 1$, and the appearance of two vacua. In the center broken phase, we expect that these two vacua are continuously connected to the two thermal equilibrium states of pure Yang-Mills theory as $\tilde{m} \rightarrow \infty$.

One crucial point here is the following. In the confined phase, the effective description of the dynamics is given in terms of the Wilson line $\Omega$ (the $b^{\prime}$-field) and dual photon $\sigma$. On the other hand, $\sigma$ is not a well-defined notion in the "deep" deconfined phase where the $S U(2)$ gauge symmetry is fully restored and the abelianization of the dynamics is lost. In other words, the combined potential (2.36) is strictly valid beyond $L \geq L_{c}(m)$ and for a range $L \lesssim L_{c}(m)$ provided the eigenvalues are sufficiently apart. For most of the range $L<L_{c}(m)$, the potential is solely in terms of $\Omega$, without $\sigma$.

Finally, we can try to perform a (very rough) extrapolation of our result to pure YangMills theory and obtain an estimate of the critical temperature of the deconfining phase transition. In the semi-classical domain, from (2.39), we find $\frac{T_{c}}{\Lambda}=\frac{1}{L_{c} \Lambda} \sim \sqrt{\frac{8 \Lambda}{m}}$, which drops with $m$, but for $m \gg \Lambda$ the result must become independent of $m$. Not much is known numerically about the decoupling scale for a Weyl fermion in the adjoint representation. In the case of $N_{c}=3$ QCD with three flavors of fundamental fermions it is known that relatively large values of the fermion mass, $m \gtrsim 5 \Lambda$, are needed in order for the phase transition to approach the deconfinement transition of the pure gauge theory [22]. Assuming that the decoupling scale for an adjoint Weyl fermion is in the range $m_{d e c} \sim(5-10) \Lambda$ we expect $T_{c} \sim(0.8-1.3) \Lambda$, broadly consistent with lattice data.

\section{Pure Yang-Mills theory}

In the previous Section, we showed that for $\tilde{m} \ll 1$ the center-symmetry restoring phase transition can be described semi-classically. In this regime the transition is driven by the competition between center-stabilizing topological molecules and center-destabilizing monopoleinstantons. In this Section, we will show that the same mechanism also exists in the pure 
gauge theory, even though in this case the effects cannot be computed reliably. This implies that it is plausible that the deconfinement transition in pure gauge theory is driven by the same topological phenomena that operate in the small $\tilde{m}$ limit.

\subsection{Non-perturbative effects on the classical background}

In this Section, we will consider possible non-perturbative contributions to the potential for the Wilson line in pure Yang Mills theory. The question is whether there are terms that favor the center-symmetric vacuum and compete with the perturbative contributions to $V(\Omega)$. We consider a classical background field on $\mathbb{R}^{3} \times \mathbb{S}^{1}$ :

$$
\Omega=\left(\begin{array}{ll}
e^{i \Delta \theta / 2} & \\
& e^{-i \Delta \theta / 2}
\end{array}\right),
$$

where $\Delta \theta$ is the separation between the eigenvalues of Wilson line. In the classical background (3.1), and at weak coupling, the Wilson line behaves as an adjoint Higgs field breaking the microscopic $S U(2)$ symmetry down to $U(1)$ at large distances. As before there are two types of elementary monopole-instantons, $\mathcal{M}_{1}$ and $\mathcal{M}_{2}$. The amplitudes associated with these instanton events are essentially the ones given by (2.11), but now without the fermion zero modes:

$$
\begin{array}{ll}
\mathcal{M}_{1}=e^{-\frac{4 \pi}{g^{2}} \Delta \theta+i \sigma} \equiv e^{-b+i \sigma}, & \overline{\mathcal{M}}_{1}=e^{-\frac{4 \pi}{g^{2}} \Delta \theta-i \sigma} \equiv e^{-b-i \sigma}, \\
\mathcal{M}_{2}=e^{-\frac{4 \pi}{g^{2}}(2 \pi-\Delta \theta)-i \sigma} \equiv \eta e^{+b-i \sigma}, & \overline{\mathcal{M}}_{2}=e^{-\frac{4 \pi}{g^{2}}(2 \pi-\Delta \theta)+i \sigma} \equiv \eta e^{+b+i \sigma} .
\end{array}
$$

The interaction between different monopole-instantons with magnetic charge $n_{m}$ and scalar charge $n_{b}$ can be computed by using the two point correlator, as in (2.22):

$$
\left\langle e^{n_{b} b+i n_{m} \sigma}(\mathbf{x}) e^{\bar{n}_{b} b+i \bar{n}_{m} \sigma}(\mathbf{y})\right\rangle_{0}=e^{-V(|\mathbf{x}-\mathbf{y}|)}=e^{-\frac{4 \pi L}{g^{2}|\mathbf{x}-\mathbf{y}|}\left(-n_{b} \bar{n}_{b}+n_{m} \bar{n}_{m}\right)} .
$$

This formula is true classically. At weak coupling, the $b$ field may acquire a radiatively induced mass. This modifies the potential as:

$$
V(|\mathbf{x}-\mathbf{y}|)=\frac{4 \pi L}{g^{2}|\mathbf{x}-\mathbf{y}|}\left(-n_{b} \bar{n}_{b} e^{-m_{b}|\mathbf{x}-\mathbf{y}|}+n_{m} \bar{n}_{m}\right)
$$

In perturbation theory, there are three possibilities for $m_{b}^{2}$ (recall that $m_{b}$ is the mass for the Wilson line, $m_{b}^{2}|\operatorname{tr} \Omega|^{2}$, obtained by studying small fluctuations around the classical vacuum): i) In supersymmetric theories with supersymmetry preserving boundary conditions, $m_{b}^{2}=0$ to all orders in perturbation theory. ii) If $m_{b}^{2}>0$, as is the case for $\mathrm{QCD}$ (adj) with periodic boundary conditions for fermions $[13,14]$, then the potential is as in (3.4). In particular, the $b$-exchange interaction is short range and has no effect on the long distance effective theory. iii) If $m_{b}^{2}<0$, as in thermal YM, then there exists an instability of the center-symmetric vacuum.

For our purposes we will consider $m_{b}=0$, because classically there is no mass gap for the holonomy fluctuations. We can now write down the effective theory for an ensemble of monopole-instantons. We get:

$$
L=\frac{1}{2} \frac{g^{2}}{(4 \pi)^{2} L}\left[\left(\partial_{i} b\right)^{2}+\left(\partial_{i} \sigma\right)^{2}\right]-\left(e^{-b}+\eta e^{+b}\right) \cos \sigma+\ldots,
$$


where we have not attempted to determine the overall coefficient of the effective potential, and we defined $b \equiv \frac{4 \pi}{g^{2}} \Delta \theta$. The ellipsis denote both perturbative and other non-perturbative contributions. The effective potential in (3.5) arises by summing the contributions of the different monopole-instantons in (3.2):

$$
\begin{aligned}
-V^{\text {n.p. }}(\Delta \theta, \sigma) & =\left(\mathcal{M}_{1}+\mathcal{M}_{2}+\overline{\mathcal{M}}_{2}+\overline{\mathcal{M}}_{2}\right) \\
& =2\left[e^{-\frac{4 \pi}{g^{2}} \Delta \theta}+e^{-\frac{4 \pi}{g^{2}}(2 \pi-\Delta \theta)}\right] \cos \sigma=4 e^{-\frac{4 \pi^{2}}{g^{2}}} \cosh b^{\prime} \cos \sigma .
\end{aligned}
$$

We would like to make a number of comments regarding this potential:

1) We note that the potential is not quite sensible as it is not manifestly periodic in $\Delta \theta$. This problem can be addressed by taking into account the existence of an infinite tower of monopole-instantons, see below.

2) We observe that the potential has an extremum at $\sigma=0$ and $\Delta \theta=0$ where the semi-classical approximation is not reliable. For the stability of the center-symmetric vacuum we must have a global minimum at $\Delta \theta=\pi$, and the sigma field must be non-tachyonic there. However, around $(\Delta \theta, \sigma)=(\pi, 0)$, and also around $(\Delta \theta, \sigma)=(\pi, \pi)$, the expansion of the potential in terms of small fluctuation yields:

$$
V^{\text {n.p. }}\left(b^{\prime}, \sigma\right)=2 e^{-\frac{4 \pi^{2}}{g^{2}}}\left\{\begin{array}{ll}
-\left(\delta b^{\prime}\right)^{2}+(\delta \sigma)^{2}+\ldots & \text { for }(\Delta \theta, \sigma)=(\pi, 0) \\
+\left(\delta b^{\prime}\right)^{2}-(\delta \sigma)^{2}+\ldots & \text { for }(\Delta \theta, \sigma)=(\pi, \pi)
\end{array} .\right.
$$

At leading order in the semi-classical expansion, the Hessian around each of the two centersymmetric saddle points is negative and we conclude that monopole-instanton effects do not favor the center-symmetric vacuum.

In the following we will address both of these points. We will argue, in particular, that topological molecules can stabilize the center.

1) Making the potential periodic: Because the $\Delta \theta$ field is an angular variable there is an infinite set of monopole-instantons on $\mathbb{R}^{3} \times \mathbb{S}^{1}$. For magnetic charge +1 , there is a tower of monopole-instantons with topological charges $Q_{T}\left(n_{w}\right)=\frac{\Delta \theta}{2 \pi}+n_{w}, n_{w} \in \mathbb{Z}$. We observe that $\mathcal{M}_{1}$ and $\overline{\mathcal{M}}_{2}$ are the $n_{w}=0$ and $n_{w}=-1$ members of this tower. Following [23] we define the generalized fugacity of the monopole-instanton amplitude as:

$$
F(\Delta \theta) e^{i \sigma}=\left[\sum_{n_{w} \in \mathbb{Z}} e^{-\frac{4 \pi}{g^{2}}\left|\Delta \theta+2 \pi n_{w}\right|}\right] e^{i \sigma},
$$

Clearly, $F(\Delta \theta+2 \pi)=F(\Delta \theta)$ is a periodic function. Using Poisson resummation it can be rewritten as:

$$
F(\Delta \theta)=\frac{1}{\pi} \sum_{n_{e} \in \mathbb{Z}} \frac{\frac{4 \pi}{g^{2}}}{\left(\frac{4 \pi}{g^{2}}\right)^{2}+n_{e}^{2}} e^{i n_{e} \Delta \theta} \equiv \frac{1}{\pi}\left(1+2 \sum_{n_{e}=1}^{\infty} c_{n_{e}} \cos n_{e} \Delta \theta\right), c_{n_{e}} \equiv \frac{\frac{4 \pi}{g^{2}}}{\left(\frac{4 \pi}{g^{2}}\right)^{2}+n_{e}^{2}},
$$

which, after using $\cos n_{e} \Delta \theta=\frac{1}{2}\left|\operatorname{tr} \Omega^{n_{e}}\right|^{2}-1$, can be expressed in terms of the gauge invariant Wilson line:

$$
F(\Omega)=\frac{1}{\pi}\left(1+\sum_{n_{e}=1}^{\infty} c_{n_{e}}\left(\left|\operatorname{tr} \Omega^{n_{e}}\right|^{2}-2\right)\right)
$$


The non-perturbatively induced potential, incorporating the entire Kaluza-Klein tower of the monopole-instantons takes the form:

$$
V^{\text {n.p. }}(\Omega, \sigma)=F(\Omega) \cos \sigma .
$$

The extremum of (3.10) is at $\Delta \theta=0, \sigma=\pi$. At this point center-symmetry is broken, gauge symmetry is restored and the abelian long distance description is invalid, exactly as for (3.6). Thus, the leading-order bosonic potential induced by monopole-instantons does not appear to stabilize center symmetry.

2) Role of topological molecules: We showed that in $\mathcal{N}=1 \mathrm{SYM}^{5}$ monopole-instantons carry fermionic zero modes and do not induce a bosonic potential. Instead, monopoleinstantons generate a fermion mass term. A bosonic potential appears at second order in the semi-classical expansion through the terms induced by composites $\left[\mathcal{M}_{\mathrm{i}} \overline{\mathcal{M}}_{\mathrm{j}}\right]$. The magnetic bion combinations $\left[\mathcal{M}_{1} \overline{\mathcal{M}}_{2}\right]+\left[\mathcal{M}_{2} \overline{\mathcal{M}}_{1}\right]$ induce a mass gap for gauge fluctuations, and the center stabilizing bions $\left[\mathcal{M}_{1} \overline{\mathcal{M}}_{1}\right]+\left[\mathcal{M}_{2} \overline{\mathcal{M}}_{2}\right]$ stabilizes the center-symmetric vacuum. We may therefore ask whether topological molecules induce similar effects in pure Yang-Mills theory.

Symmetry permits, at second order in the semi-classical expansion, terms of the form:

$$
-V^{(2)}(b, \sigma)=c_{1} \eta \cos 2 \sigma+c_{2}\left(e^{-2 b}+\eta^{2} e^{2 b}\right)+c_{3}\left(e^{-2 b}+\eta^{2} e^{2 b}\right) \cos 2 \sigma+c_{4} \eta
$$

The terms in (3.11), which can be made periodic in $b$ by a procedure similar to the one that led to (3.10), can be thought as due to molecular monopole-instantons of the form:

$$
\begin{aligned}
& {\left[\mathcal{M}_{1} \overline{\mathcal{M}}_{2}\right] \sim e^{+2 i \sigma}, \quad\left[\mathcal{M}_{2} \overline{\mathcal{M}}_{1}\right] \sim e^{-2 i \sigma},} \\
& {\left[\mathcal{M}_{1} \overline{\mathcal{M}}_{1}\right] \sim e^{-2 b}, \quad\left[\mathcal{M}_{2} \overline{\mathcal{M}}_{2}\right] \sim \eta^{2} e^{+2 b},} \\
& {\left[\mathcal{M}_{1} \mathcal{M}_{2}\right] \sim \eta, \quad\left[\overline{\mathcal{M}}_{1} \overline{\mathcal{M}}_{2}\right] \sim \eta} \\
& {\left[\mathcal{M}_{1} \mathcal{M}_{1}\right] \sim e^{-2 b+2 i \sigma}, \quad\left[\overline{\mathcal{M}}_{1} \overline{\mathcal{M}}_{1}\right] \sim e^{-2 b-2 i \sigma},} \\
& {\left[\mathcal{M}_{2} \mathcal{M}_{2}\right] \sim \eta^{2} e^{+2 b-2 i \sigma}, \quad\left[\overline{\mathcal{M}}_{2} \overline{\mathcal{M}}_{2}\right] \sim \eta^{2} e^{+2 b+2 i \sigma} .}
\end{aligned}
$$

In Section 2.3.2, we showed that there are SUSY and softly broken SUSY theories in which we can reliably demonstrate that these molecules generate a center-symmetric minimum. Below we will demonstrate, using the BZJ prescription, that topological molecules also stabilize the center in pure YM theory. Clearly, in order for the second order terms in (3.11) to be more important than the leading-order term in (3.10), we have to push the expansion beyond the semi-classical domain. We will therefore not attempt to perform a quantitative calculation. Our goal is to show that the same mechanism that drives the center-symmetry changing transition in the softly broken $\mathcal{N}=1$ theory also operates in pure Yang-Mills theory.

\subsubsection{Quasi-zero modes and bion amplitudes}

In this Section, we will study the amplitude of topological molecules in the pure Yang-Mills theory on the classical background. The amplitudes for the molecular monopole-instanton

\footnotetext{
${ }^{5}$ The same is true in $\mathcal{N}=2$ Seiberg-Witten theory softly broken to $\mathcal{N}=1$.
} 
events can be found by integrating over the relative separation quasi-zero mode between its constituents. This will be similar to the study in Section 2.3.2, where we performed an analysis for the case of $n_{f}$ adjoint fermions, where $n_{f}=1$ corresponds to $\mathcal{N}=1$ SYM theory. The result for pure Yang Mills theory can be obtained by taking the limit $n_{f}=\epsilon \rightarrow 0$ in (2.26), (2.29), and (2.30).

We begin with topological molecules of the type $\left[\mathcal{M}_{1} \overline{\mathcal{M}}_{2}\right]$. The integral over the quasizero mode is the same as in (2.26), except that we have to take the $\epsilon \rightarrow 0$ limit. We find

$$
I(\lambda, \epsilon)=\lambda^{4 \epsilon-3} \Gamma(4 \epsilon-3)=\lambda^{-3}\left(-\frac{1}{24 \epsilon}+\frac{1}{6}\left(-\log (\lambda)+\gamma-\frac{11}{6}\right)+O(\epsilon)\right) .
$$

The divergence in the $\epsilon \rightarrow 0$ limit is due to over-counting of uncorrelated monopole-instanton events, which are already included in the dilute monopole-instanton gas approximation. In theories with fermions, this long-distance divergence is cut-off by the fermion zero mode exchange, both in quantum mechanical examples [21] and in quantum field theories [19, 23]. In theories without fermions, in order not to double-count, this divergence needs to be subtracted, see $[16,18]$ for a quantum mechanical example. Consequently, the prefactor of the magnetic bion amplitude is:

$$
c_{1}(g)=\frac{2 \pi a^{2}}{3}\left(\frac{8 \pi}{g^{2}}\right)^{3}\left(-\log \left(\frac{g^{2}}{8 \pi}\right)+\gamma-\frac{11}{6}\right)
$$

where $a$ is the coefficient of the one monopole-instanton amplitude, which is set to one in (3.2) and which can be restored if desired.

Next, we consider the neutral bions $\left[\mathcal{M}_{1} \overline{\mathcal{M}}_{1}\right]$. Here, the constituents interact attractively both due to $\sigma$ and $b$-exchange, and we need to apply the BZJ prescription. The result is:

$$
c_{2}(g)=\frac{2 \pi a^{2}}{3}\left(-\frac{8 \pi}{g^{2}}\right)^{3}\left(-\log \left(-\frac{g^{2}}{8 \pi}\right)+\gamma-\frac{11}{6}\right)=-c_{1}(g) \pm(i \pi) \frac{2 \pi a^{2}}{3}\left(\frac{8 \pi}{g^{2}}\right)^{3} .
$$

In analogy with the quantum mechanics example where the ambiguity associated with nonBorel summability of the perturbation theory is canceled by the molecular instanton-antiinstanton contribution $[16,17]$, we also expect the ambiguity associated with the non-Borel summability of the pure YM theory on $\mathbb{R}^{3} \times S^{1}$ to be canceled by the two-fold ambiguity of the amplitude for neutral bions. This implies that the imaginary part in (3.14) must cancel by large-orders in perturbation theory, and we discard that term. The remaining term in (3.15) satisfies

$$
c_{2}(g)=-c_{1}(g) .
$$

For a more detailed discussion of this conjecture and the available theoretical evidence in its favor, see [19].

Next, consider the $\left[\mathcal{M}_{1} \mathcal{M}_{1}\right]$ and $\left[\mathcal{M}_{2} \mathcal{M}_{2}\right]$ amplitudes. Now, $\sigma$ exchange is repulsive and $b$ exchange is attractive, leading to a cancellation. This means that the self-dual monopoleinstanton events are not correlated, and the molecular amplitude vanishes, $c_{3}(g)=0$. The same conclusion is also obtained in Ref. [24]. 
For the $\left[\mathcal{M}_{1} \mathcal{M}_{2}\right]$ amplitude, slightly more care is needed. The interaction again cancels between attractive $\sigma$ exchange and repulsive $b$ exchange. This would seem to imply $c_{4}(g)=0$, as in the previous case. However, $\left[\mathcal{M}_{1} \mathcal{M}_{2}\right]$ molecules with sizes less than the compactification scale correspond to ordinary BPST-instantons. Taking into account small 4d instantons corresponds to $c_{4}(g) \sim \eta$. Large $4 \mathrm{~d}$ instantons do not exist because large uncorrelated $\left[\mathcal{M}_{1} \mathcal{M}_{2}\right]$ molecules are already included in the instanton-monopole contribution. This implies that there is a finite instanton term, but since the $4 \mathrm{~d}$-instanton does not depend on the $b$ and $\sigma$ field we find that the leading semi-classical instanton contribution only enters as a constant term in the effective action.

Combining the terms (3.14), (3.15), and (3.16) that appear at second order in the semiclassical expansion we find:

$$
V^{(2)}(b, \sigma)=-c_{1} \eta \cos 2 \sigma+c_{1}\left(e^{-2 b}+\eta^{2} e^{2 b}\right) .
$$

This is indeed the same result as in (softly broken) $\mathcal{N}=1$ supersymmetric theory. The combined potential of monopole-instantons and bions is

$$
V^{(1)}(b, \sigma)+V^{(2)}(b, \sigma)=-\eta^{1 / 2} \cosh b^{\prime} \cos \sigma-c_{1} \eta \cos 2 \sigma+c_{1} \eta \cosh 2 b^{\prime},
$$

leading to:

$$
\begin{aligned}
& \tilde{m}_{b^{\prime}}^{2}=\left[-\eta^{1 / 2}+4 c_{1} \eta\right], \\
& \tilde{m}_{\sigma}^{2}=\left[\eta^{1 / 2}+4 c_{1} \eta\right] .
\end{aligned}
$$

Thus, we find that second-order effects in the semi-classical expansion, in particular neutral bions, stabilize the center symmetry, without destabilizing the $\sigma$ mode. As in the discussion around (3.9) and (3.10), in order to make periodicity of the potential manifest, we may sum over the Kaluza-Klein tower of the neutral bions. This leads to a center-stabilizing potential for the Wilson line, given by $V_{\mathrm{b}-\text { tower }}(\Omega) \sim \sum_{n}\left|\operatorname{tr} \Omega^{n}\right|^{2}$, similar to (3.9). However, as stated earlier, the stabilization of center-symmetry by the neutral bion induced potential requires strong coupling where neutral bion term can overcome the monopole-instanton induced instability as well as the perturbatively induced potential. In this regime, we cannot perform a quantitative calculation. It is nevertheless intriguing that the same effect that leads to center stabilization at weak coupling for softly broken $\mathcal{N}=1$ theory is present in pure Yang Mills theory as well. For an attempt to connect semi-classical neutral bion molecules to strong coupling effects and infrared renormalons, see the recent work [19].

\subsubsection{Connecting monopole-instantons to dyon particles}

The neutral bion induced potential (3.17), just like the monopole-instanton induced potential, is not manifestly periodic in $\Delta \theta$. This problem, as in the case of monopole-instantons, can be addressed by taking into account the whole Kaluza-Klein tower of neutral bion molecules. 
In this Section, we will show that the tower of monopole-instantons can be interpreted, via Poisson resummation, as the contribution from dyon particles. ${ }^{6}$

Consider the Poisson duality relation for the tower of monopole-instantons, see (3.9):

$$
\left[\sum_{n_{w} \in \mathbb{Z}} e^{-\frac{4 \pi}{g^{2}}\left|\Delta \theta+2 \pi n_{w}\right|}\right] e^{i \sigma}=\left[\frac{1}{\pi} \sum_{n_{e} \in \mathbb{Z}} \frac{\frac{4 \pi}{g^{2}}}{\left(\frac{4 \pi}{g^{2}}\right)^{2}+n_{e}^{2}} e^{i n_{e} \Delta \theta}\right] e^{i \sigma}
$$

The phase $e^{i n_{e} L A_{4}}=e^{i n_{e} \Delta \theta}$ is the canonical coupling of a charged particle to a background gauge field $A_{4}$, and $e^{i \sigma}$ is a $3 \mathrm{~d}$ instanton amplitude associated with magnetic charge one.

Consider a massive particle on $\mathbb{R}^{3,1}$ with magnetic and electric charge $\left(n_{m}, n_{e}\right)$ and mass $M_{\left(n_{m}, n_{e}\right)}$, called a dyon. By Gauss' law, there is a combined electric and magnetic flux coming out of this particle;

$$
\int_{\mathbb{S}^{2}=\partial \mathbb{R}^{3}}(\vec{E}+i \vec{B}) \cdot d \vec{\Sigma}=n_{e}+i \frac{4 \pi}{g^{2}} n_{m} \equiv q_{e}+i q_{m},
$$

where $\mathbb{S}^{2}=\partial \mathbb{R}^{3}$ is a sphere at infinity. We can analytically continue to Euclidean time (consider the theory on $\mathbb{R}^{4}$ ) and then compactify one of the directions, i.e., consider the theory on $\mathbb{R}^{3} \times \mathbb{S}^{1}$.

A dyon particle whose world-line wraps the $\mathbb{S}^{1}$ corresponds to a finite action instanton, $S_{\left(n_{m}, n_{e}\right)}=L M_{\left(n_{m}, n_{e}\right)}$. The amplitude associated with the dyon is,

$$
e^{-S_{\left(n_{m}, n_{e}\right)}} e^{i n_{e} \theta_{e}+i n_{m} \theta_{m}} \equiv e^{-L M_{\left(n_{m}, n_{e}\right)}} e^{i n_{e} \theta_{e}+i n_{m} \sigma},
$$

where $e^{i \theta_{e}}$ and $e^{i \theta_{m}}$ are electric and magnetic Wilson lines. The magnetic Wilson line is naturally interpreted in terms of the dual photon ${ }^{7} \sigma$. The duality relation (3.21) therefore admits an interesting interpretation: its right hand side may be re-written as a sum over massless/light dyons with charges $\left(n_{m}, n_{e}\right)=\left(1, n_{e}\right)$ whose world-lines wrap around the $\mathbb{S}^{1}$ :

$$
\frac{1}{\pi} \sum_{n_{e} \in \mathbb{Z}} \frac{q_{m}}{q_{m}^{2}+q_{e}^{2}} e^{-L M\left(q_{m}, q_{e}\right)} e^{i n_{e} \theta_{e}+i \sigma} .
$$

\footnotetext{
${ }^{6}$ A number of authors, see, e.g., [20] and references therein, refer to the monopole-instantons $\mathcal{M}_{1}$ and $\mathcal{M}_{2}$ as "dyons", because they are magnetically charged and self-dual. We believe that this choice of words is not quite appropriate. The amplitude of a monopole-instanton has the form $e^{-b+i \sigma} \sim e^{-A_{4}+i \sigma}$. However, a dyon particle with both electric and magnetic charge couples to $\left(A_{4}, \sigma\right)$ as $e^{i q_{e} A_{4}+i q_{m} \sigma}$, in both Minkowski and Euclidean space. We will see that the Kaluza-Klein tower of monopole-instantons can be written as a sum over dyons that exhibit the expected coupling for electrically and magnetically charged particles.

${ }^{7}$ We can see this starting from abelian duality in $4 \mathrm{~d}$, then compactify the theory on $\mathbb{R}^{3} \times \mathbb{S}^{1}$, and finally match the result to $3 \mathrm{~d}$ abelian duality. The abelian duality on $4 \mathrm{~d}$ is expressed by $F_{\mu \nu}^{D}=\frac{1}{2} \epsilon_{\mu \nu \rho \sigma} F_{\mu \nu}$ where $F_{\mu \nu}=\partial_{\mu} A_{\nu}-\partial_{\nu} A_{\mu}$ and $F_{\mu \nu}^{D}=\partial_{\mu} A_{\nu}^{D}-\partial_{\nu} A_{\mu}^{D}$, where $A_{\mu}, A_{\mu}^{D}$ are the original and the dual gauge potential. Using dimensional reduction and splitting the duality relation into $4 i$ and $i j$ components, we obtain $\partial_{i} A_{4}^{D}=$ $-\frac{1}{2} \epsilon_{i j k} F_{j k}$ and $F_{i j}^{D}=\epsilon_{i j k} \partial_{k} A_{4}$. The first relation is the well-known abelian duality relation in $3 \mathrm{~d}$, where we identify $A_{4}^{D}=\sigma \equiv \theta_{m}$ and $A_{4}=\sigma^{D}=\theta_{e}$. The monopole-instanton amplitude is naturally expressed in terms of $\theta_{m}=\sigma$ and $\theta_{e} \equiv \Delta \theta$.
} 
In the classical background, the long distance dynamics is abelian and the sum over monopoleinstanton amplitudes maps, via Poisson resummation, to a sum over the electric charges of the dyons.

From this point of view, we find (3.21) quite intriguing. This relation makes perfect sense in $\mathcal{N}=2$ Seiberg-Witten theory [23]. In that context, on $\mathbb{R}^{4}$, the theory has a classical moduli space along which gauge symmetry is reduced to $U(1)$ due to adjoint Higgsing by the vev $\langle\Phi\rangle=v T^{3}$. In the semi-classical domain of the $\mathcal{N}=2$ theory, the Poisson duality relation is reliable. Moreover, in $\mathcal{N}=2$ theory, the combination $\int_{\mathbb{S}^{2}} v(\vec{E}+i \vec{B}) \cdot d \vec{\Sigma}=Z_{\left(n_{m}, n_{e}\right)}$ appears as the central charge in the supersymmetry algebra.

In pure Yang-Mills theory, the combination of electric and magnetic charges $\int_{S^{2}}(\vec{E}+i \vec{B})$. $d \vec{\Sigma}$ (without the vev) appears naturally. However, we also know that there is no apparent adjoint Higgsing on $\mathbb{R}^{4}$ and it seems hard to make sense out of the Poisson duality relation in that case. One can speculate that there may be a connection between Poisson duality and the maximal abelian gauge proposal by 't Hooft [25], which is based on using a specific gauge condition applied to a composite operator to define an adjoint Higgs field, and the corresponding abelianized $S U(N) \rightarrow U(1)^{N-1}$ theory. In that case, of course, monopoles (as well as dyons) appear. However, the theory is strongly coupled, and the abelian dynamics is not well understood. This is a direction worthy of further pursuit.

\section{Comments on the literature}

In this Section, we will attempt to clarify the relation between our work and previous analytical approaches to the deconfinement transition in the literature.

The question whether one can study the deconfinement phase transition at weak coupling was considered by Aharony et al. in [26]. These authors found a realization of this idea in a finite spatial volume $\mathbb{S}^{3} \times \mathbb{S}^{1}$, but rejected the possibility that an example can be found for an infinite spatial volume, in particular on $\mathbb{R}^{3} \times \mathbb{S}^{1}$. Ref. [26] studied large- $N$ (strictly, $N=\infty$ ) pure Yang-Mills theory on small $\mathbb{S}^{3} \times \mathbb{S}^{1}$ by integrating out the perturbatively weakly coupled modes and thus mapping the field theory to a matrix model. This approach pushes the deconfinement transition to the weak coupling regime. However, since they study gauge theory on a small sphere $\mathbb{S}^{3}$, approaching the thermodynamic limit requires taking the infinite- $N$ limit. In particular, the approach of [26] does not apply to gauge theories of finite rank.

A way around the obstacle of analytical "intractability" of deconfinement in an infinite spatial volume was found in Refs. $[27,28]$ by compactifying the gauge theory on $\mathbb{R}^{2} \times \mathbb{S}_{L}^{1} \times \mathbb{S}_{\beta}^{1}$ where $\mathbb{S}_{L}^{1}$ is a spatial circle and $\mathbb{S}_{\beta}^{1}$ is the thermal circle. The size of the spatial circle provides a tunable control parameter, the counter-part of $\mathbb{S}^{3}$ in the approach of [26]. The advantage of this formalism is that the small volume theory is still a field theory in an infinite spatial volume (as opposed to a matrix model), although it is $\mathbb{R}^{2}$ and not $\mathbb{R}^{3}$. The approach of $[27,28]$ also works for finite rank gauge theories, mapping the deconfinement transition to a phase transition in two-dimensional spin systems; the relevant spin systems are the affine XY-spin models with symmetry breaking perturbations. 
In the present paper, we gave a reliable semi-classical analysis of the center-symmetry changing phase transition on $\mathbb{R}^{3} \times \mathbb{S}^{1}$. The use of semi-classical methods in connection with the center-symmetry changing transition was previously investigated in a series of papers by Diakonov and collaborators [20,24, 29,30]:

1) The first of these papers, a review published in 2002 [29], suggests that centersymmetry can be stabilized by monopole-instantons (dyons, in the language of Diakonov et al.). Diakonov obtains the term $V \sim \cosh \frac{4 \pi}{g^{2}}(\pi-\Delta \theta)$ in the monopole-instanton induced potential, see (2.33) and (3.6), and observes that this contribution has a minimum at the center symmetric point $\Delta \theta=\pi$. However, this conclusion is based on neglecting the the $\cos \sigma$ term which arises from the coupling to the dual photon. Indeed, we found that in the semi-classical case $m \ll \Lambda$ monopole-instantons favor center-symmetry breaking.

2) $\mathcal{N}=1 \mathrm{SYM}$ theory on $\mathbb{R}^{3} \times \mathbb{S}^{1}$ is presented as an example for center stabilization by monopole-instantons in Refs. [20,24,29]. This interpretation is common in the literature but, as we showed above, it is physically not correct. Monopole-instantons in $\mathcal{N}=1 \mathrm{SYM}$ have fermion zero modes, and they do not generate a bosonic potential for the Wilson line. Rather, they generate a fermion bilinear which determines the superpotential. The bosonic potential can be found via supersymmetry using $V \sim\left|\frac{\partial W}{\partial b}\right|^{2}$, as in Section 2.2, or using the BZJ-prescription, as in Section 2.3. In either case the conclusion is that the physical mechanism generating the potential for the Wilson line is related to topological molecules.

3) A quantitative theory of a confining ensemble of monopole-instantons was proposed in [24]. The paper argues that one can analyze the vacuum of Yang-Mills theory by semiclassical means, and that, furthermore, the only topological configurations that contribute to the non-perturbative potential for the gauge holonomy satisfy: i) the self-duality condition, and ii) a (magnetic) charge neutrality constraint. This means that, contrary to item 1), instanton-monopoles $\left[\mathcal{M}_{1}\right],\left[\mathcal{M}_{2}\right]$ and $\left[\overline{\mathcal{M}}_{1}\right],\left[\overline{\mathcal{M}}_{2}\right]$ are excluded. The self-dual neutral objects are KvBLL-calorons $[8,9]$, 4d BPST instantons at finite temperature and non-zero holonomy, and multi-calorons. Calorons can be viewed as topological molecules of the form $\left[\mathcal{M}_{1} \mathcal{M}_{2}\right]$ and $\left[\overline{\mathcal{M}}_{1} \overline{\mathcal{M}}_{2}\right]$. We observed in (3.12) that at the classical level there is no coupling of instantons to the holonomy. The claim in Ref. [24] is that a potential for the Polyakov line can arise from the collective coordinate measure. This contribution is formally of higher order in the coupling constant.

In the pure gauge theory the critical temperature for deconfinement is of order $\Lambda$, and it is clear that there cannot be a systematic semi-classical theory of the transition. The continuity argument outlined in Figure 1 provides a less ambitious program: We connect the strongly coupled center-symmetry changing phase transition in Yang-Mills theory to a semi-classical phase transition in mass-perturbed super-Yang-Mills theory on $\mathbb{R}^{3} \times \mathbb{S}^{1}$. The semi-classical calculation at small $m$ provides two important lessons: a.) Both monopole-instantons (selfdual and magnetically charged objects ${ }^{8}$ ) and neutral bions (non-self dual and magnetically

\footnotetext{
${ }^{8}$ It is clear that there cannot be a general argument that rules out contributions from magnetically charged objects. In particular, Polyakov's solution to the Yang-Mills adjoint Higgs system on $\mathbb{R}^{3}$ [31] and the solution of deformed Yang-Mills theory [32] on $\mathbb{R}^{3} \times \mathbb{S}^{1}$ map the gauge theory partition function to a grand canonical ensemble of magnetic charges.
} 
neutral objects like $\left.\left[\mathcal{M}_{1} \overline{\mathcal{M}}_{1}\right]\right)$ contribute to the potential for the gauge holonomy and $b$.) these are the leading contributions to the potential in the controllable small- $L, m$ regime. Thus, we believe that our results contradict the assumptions in [24].

Finally, we also note that detailed phenomenological studies of the effective lagrangian for the Polyakov line in pure Yang-Mills theory were carried out by Pisarski and collaborators [33-36]. Center-stabilizing double-trace operators are considered in Ref. [32] to address a semi-classical mechanism of confinement and large- $N$ volume independence, and in Ref. [37] to study phases with partial center symmetry breaking. The present study provides a microscopic explanation of the origin of the center-stabilizing double-trace terms in these studies.

\section{Conclusions and outlook}

In this paper, we argued that the center-symmetry changing phase transition in thermal Yang Mills theory is continuously connected to the phase transition in softly broken $\mathcal{N}=1$ theory on $\mathbb{R}^{3} \times \mathbb{S}^{1}$. We showed that for small values of the adjoint fermion mass $m$ the critical scale $L_{c}$ is analytically calculable. We also provided theoretical evidence that the same mechanism that drives the phase transition at small $m$, the competition between center-stabilizing topological molecules and center-destabilizing monopole-instantons, also exists in pure Yang Mills theory.

There are a number of directions worthy of further pursuit, including both numerical and analytical studies:

1. The phase diagram in Figure 1 can be explored with lattice methods that are available today. Furthermore, lattice simulations can be used to study the role of neutral non-selfdual topological defects (such as the neutral bions), for example, by using the techniques of Ref. [38].

2. One may generalize the semi-classical study of center-symmetry changing phase transition on $\mathbb{R}^{3} \times \mathbb{S}^{1}$ to all gauge groups, exploring the role of various topological excitations in the symmetry realization and the nature of the phase transition.

Let us also make a few comments on the $S U(N), N \geq 3$ case. Here, there are $N$-types of monopole-instantons, associated with the $(N-1)$ simple roots and one affine root. As in the $S U(2)$ theory, in the softly broken $\mathcal{N}=1$ theory, these monopole-instantons, as well as perturbative one-loop fluctuations, tend to break center-symmetry. There are $N$-types of magnetic bions and $N$-types of neutral bions. The magnetic bions, for all $N \geq 3$, unlike the $N=2$ case, lead to a center-destabilizing potential. As in the $S U(2)$ case, for all $N \geq 3$, the neutral bions lead to a center-stabilizing potential. For $N \geq 3$ we found a first order phase transition consistent with lattice gauge theory results.

Finally, we note that the dependence of $T_{c}$ on the topological angle $\theta$ can also be studied within our approach, resulting in a decrease of $T_{c}$, in agreement with recent lattice studies [39]. Details of the calculations including $\theta$-dependence and higher rank groups will be given elsewhere. 


\section{Acknowledgments}

We thank Philip Argyres and Larry Yaffe for useful discussions and João Penedones for correspondence on the manuscript. E.P. thanks SFSU, KIAS, and APCTP for hospitality during the completion of this paper and is indebted to Piljin Yi and Yoon Pyo Hong for many useful discussions. We thank the referee for pointing out the non-cancellation of the nonzero mode determinants discussed in the appendix. This work was supported in part by the US Department of Energy grant DE-FG02-03ER41260 (T.S.) and the National Science and Engineering Research Council of Canada (E.P.).

\section{A. Supersymmetry and the non-cancelling nonzero-mode determinants}

In this work, we have derived the effective lagrangian for the Polyakov line and the dual photon by expanding around the supersymmetric limit of a massless Weyl fermion. In deriving equ. $(2.14,2.33)$, we have used the cancellation of nonzero mode determinants in the $N=1$ supersymmetric theory. This cancellation has also been used in many other instances in the literature, for example in the calculation of the exact superpotential and gluino condensate in $N=1$ supersymmetric gauge theory on $\mathbb{R}^{3} \times \mathbb{S}^{1}[12,40]$.

In this Appendix we discuss an important issue that has not received proper attention within the context of $N=1$ supersymmetric theories on $\mathbb{R}^{3} \times \mathbb{S}^{1}$ — the fact that the nonzero mode determinants in the field of a monopole do not precisely cancel. While we will argue that this phenomenon has no significant effect on our main result, we include this discussion for completeness, as it fills a gap in the literature on $N=1$ theories on $\mathbb{R}^{3} \times \mathbb{S}^{1}$.

The point is that in the supersymmetric theory, the determinants of nonzero mode fluctuations around the BPS or KK monopole-instantons on $\mathbb{R}^{3} \times \mathbb{S}^{1}$ do not precisely cancel, despite the fact that the solutions preserve one-half of the supersymmetry. This noncancellation occurs essentially because of the slow fall-off of the monopole-instanton background at infinity in $\mathbb{R}^{3}[42,43]$. The nonvanishing of the nonzero mode determinants around supersymmetric monopole-instanton backgrounds in $N=1$ theories on $\mathbb{R}^{3} \times \mathbb{S}^{1}$ is the $N=1$ counterpart of the mass and central-charge renormalization ${ }^{9}$ of BPS monopoles in $4 \mathrm{~d} N=2$ supersymmetric theories. As we will see, the non-cancellation of the nonzero mode determinants is perfectly consistent and is, in fact, required by supersymmetry and holomorphy.

In the context of purely $3 \mathrm{~d}$ theories with extended supersymmetry, obtained by reducing $N=24 \mathrm{~d}$ theories, this non-cancellation has been known since [41]. However, it was not addressed in the original calculation of nonperturbative effects in $N=1$ theories on $\mathbb{R}^{3} \times \mathbb{S}^{1}$ $[12,40]$. Only recently, a relevant calculation on $\mathbb{R}^{3} \times \mathbb{S}^{1}$ appeared in the literature [45], in the context of theories with $N=2$ supersymmetry. While $N=2$ theories share many features with the $N=1$ theory of interest to us, the most important difference is that the branch of moduli space considered in [45] is the $4 \mathrm{~d}$ Coulomb branch, where the abelianization of the gauge group is due to a nonzero expectation value $|a|$ of the adjoint Higgs matter supermultiplet. In particular, $|a| \gg \Lambda_{N=2}$ was required for consistency of the calculation

\footnotetext{
${ }^{9}$ See [44] for a recent review and references.
} 
of [45] (while an arbitrary $|a| L$ was allowed). In contrast, we are interested in the $N=1$ theory where the adjoint Higgs field is absent and the abelianization is due, instead, to a Wilson line expectation value. Thus, the result of [45] is not directly applicable to the case of interest to us.

From the discussion in the previous paragraph, it is clear that a calculation relevant for $N=1$ theories on $\mathbb{R}^{3} \times \mathbb{S}^{1}$ is not present in the literature. In what follows, we shall perform this calculation. The novel ingredient that we will use is the Nye-Singer index theorem on $\mathbb{R}^{3} \times \mathbb{S}^{1}[10]$ in backgrounds with nontrivial holonomy, in the form studied by two of us (EP and MÜ) in [11]. We will also benefit from insight gained from refs. [41, 45, 49].

The main object of interest turns out to be the "index" $I\left(M^{2}\right)$, defined ${ }^{10}$ as:

$$
I\left(M^{2}\right)=\operatorname{tr} \frac{M^{2}}{\Delta_{-}+M^{2}}-\operatorname{tr} \frac{M^{2}}{\Delta_{+}+M^{2}},
$$

where $\Delta_{-}=D^{\dagger} D=-D_{\mu} D^{\mu}-\frac{1}{2} \sigma_{\mu \nu} F^{\mu \nu}$ and $\Delta_{+}=D D^{\dagger}=-D_{\mu} D^{\mu}$, where $D$ is the Weyl operator and $D_{\mu}$ - the covariant derivative in the monopole-instanton background. In a selfdual monopole-instanton self-dual background, it is the operator $\Delta_{-}$that has zero modes; see, e.g., [46] for a review of this notation. To establish a relation between $I\left(M^{2}\right)$ and the nonzero mode determinants, we note the identity:

$$
\int_{\mu^{2}}^{\Lambda_{P V}^{2}} \frac{d M^{2}}{M^{2}} I\left(M^{2}\right)=\operatorname{tr} \ln \frac{\Delta_{+}+\mu^{2}}{\Delta_{+}+\Lambda_{P V}^{2}}-\operatorname{tr} \ln \frac{\Delta_{-}+\mu^{2}}{\Delta_{-}+\Lambda_{P V}^{2}}=\ln \operatorname{det} \frac{\Delta_{+}+\mu^{2}}{\Delta_{+}+\Lambda_{P V}^{2}} \frac{\Delta_{-}+\mu^{2}}{\Delta_{-}+\Lambda_{P V}^{2}},
$$

where $\Lambda_{P V}$ is the Pauli-Villars mass and $\mu$ is an auxiliary parameter which will be eventually taken to zero. We can now use (A.2) to define:

$$
R=\lim _{\mu \rightarrow 0}\left(\mu^{4} e^{\int_{\mu^{2}}^{\Lambda_{P}^{2}} \frac{d M^{2}}{M^{2}} I\left(M^{2}\right)}\right)^{\frac{3}{4}}=\left(\frac{\operatorname{det} \Delta_{+}}{\operatorname{det}^{\prime} \Delta_{-}} \frac{\operatorname{det} \Delta_{-}+\Lambda_{P V}^{2}}{\operatorname{det} \Delta_{+}+\Lambda_{P V}^{2}}\right)^{\frac{3}{4}} .
$$

Here, "det'" is the determinant with the zero modes omitted, i.e., $\operatorname{det}^{\prime} \Delta_{-} \equiv \lim _{\mu \rightarrow 0} \frac{\operatorname{det} \Delta_{-}+\mu^{2}}{\mu^{4}}$, using the fact that $\Delta_{-}$has two zero modes. The quantity $R$ of (A.3) is, as is made clear from the second identity, equal to the ratio of nonzero modes' determinants around monopoleinstanton backgrounds appearing in the $N=1$ theory. Notice also that $R$ is equal to the fluctuation determinant [45] for the $N=2$ theory to the power of $3 / 2$. The various contributions making up the ratio in (A.3) are as follows: the adjoint fermion contribution is $\left(\operatorname{det}^{\prime} \Delta_{-} \operatorname{det} \Delta_{+}\right)^{\frac{1}{4}}$, while, in a $4 \mathrm{~d}$ background Lorentz gauge, the gauge field determinant is $\left(\operatorname{det}^{\prime} \Delta_{-}\right)^{-1}$ and the ghost determinant- $\left(\operatorname{det} \Delta_{+}\right)^{\frac{1}{2}}$, see [46] for details.

\footnotetext{
${ }^{10}$ We use quotation marks, since the value of the function $I(0)$ is, indeed, the index of the adjoint Dirac operator in a monopole-instanton background, but the quantity $I\left(M^{2}\right)$ itself depends on $M^{2}[42]$.
} 
Our main goal here is to compute the ratio of determinants $R$ (A.3). We will use the expression for $I\left(M^{2}\right)$ from [11]:

$$
\begin{aligned}
I\left(M^{2}\right) & =I_{1}+I_{2}\left(M^{2}\right)=\frac{2 L v}{\pi}+\sum_{p=-\infty}^{\infty}\left(\frac{\frac{2 \pi p}{L}+v}{\left|\left(\frac{2 \pi p}{L}+v\right)^{2}+M^{2}\right|^{\frac{1}{2}}}-\frac{\frac{2 \pi p}{L}-v}{\left|\left(\frac{2 \pi p}{L}-v\right)^{2}+M^{2}\right|^{\frac{1}{2}}}\right), \\
I_{1} & \equiv \frac{2 L v}{\pi} .
\end{aligned}
$$

The definition of $I_{2}\left(M^{2}\right)$ is evident; see also (A.6) below. The relation between $v$, the expectation value of $A_{4}$, and $\Delta \theta$, the angular distance between the eigenvalues of the Polyakov loop, is:

$$
\Delta \theta=L v \in(0,2 \pi)
$$

We note that $\frac{2 L v}{\pi}=4 Q$, where $Q$ is the topological charge of the solution, which equals $1 / 2$ at the center symmetric point $v=\frac{\pi}{L}$.

If $L$ is set to zero and the $p$-sums restricted to $p=0$, this is exactly the quantity $I\left(M^{2}\right)_{\mathbb{R}^{3}}$, found in [42], and yielding the well-known result $I(0)=2$ for the adjoint representation. Note that the function $I\left(M^{2}\right)_{\mathbb{R}^{3}}$ played a role in both [41], where it was used directly, and [45], where the related difference between the density of states of $\Delta_{-}$and $\Delta_{+}$, see [43], was used. The novelty on $\mathbb{R}^{3} \times \mathbb{S}^{1}$ is that a sum over Kaluza-Klein modes and a contribution of the topological charge to the index (the non integer $I_{1}$ term in (A.4)) appear. The first term in (A.4) is the bulk contribution to the index and the second - the surface term. The surface term is given by the Kaluza-Klein sums in (A.4), defined using zeta-function regularization; in fact, the second term in (A.4),

$$
I_{2}\left(M^{2}\right)=\sum_{p=-\infty}^{\infty}\left(\frac{\frac{2 \pi p}{L}+v}{\left|\left(\frac{2 \pi p}{L}+v\right)^{2}+M^{2}\right|^{\frac{1}{2}}}-\frac{\frac{2 \pi p}{L}-v}{\left|\left(\frac{2 \pi p}{L}-v\right)^{2}+M^{2}\right|^{\frac{1}{2}}}\right),
$$

as shown in detail in [11], leads to:

$$
I_{2}(0)=\sum_{p=-\infty}^{\infty}\left(\operatorname{sign}\left(\frac{2 \pi p}{L}+v\right)-(v \rightarrow-v)\right)=-\frac{2 L v}{\pi}-2\left\lfloor-\frac{v L}{2 \pi}\right\rfloor+2\left\lfloor\frac{v L}{2 \pi}\right\rfloor,
$$

where $\lfloor x\rfloor$ is the largest integer smaller than $x$. Thus, the topological charge contribution to the index, $I_{1}$, is canceled by the non integer contribution from the $\mathrm{KK}$ sum, $I_{2}(0)$, and the index equals 2 for $0<v L<2 \pi$.

\section{A.1 Calculating the ratio of nonzero mode determinants}

In the previous Section, we showed that $I_{2}(0)=2$. Here, we use the expression for $I_{2}\left(M^{2}\right)$ to compute the ratio of non-zero mode determinants $R$. We begin with the expression for $R$ given in (A.3), multiplied by $e^{-S_{0}}=e^{-\frac{4 \pi v L}{g^{2}\left(\Lambda_{P V}\right)}}$, where $S_{0}$ is the classical action of a BPS monopole:

$$
e^{-S_{0}} R=e^{-S_{0}} \lim _{\mu \rightarrow 0}\left(\mu^{4} e^{I_{1} \log \frac{\Lambda_{P V}^{2}}{\mu^{2}}} e^{\int_{\mu^{2}}^{\Lambda_{P}^{2}} \frac{I_{2}\left(M^{2}\right)}{M^{2}} d M^{2}}\right)^{\frac{3}{4}}
$$




$$
\begin{gathered}
=e^{-\frac{4 \pi v L}{g^{2}\left(\Lambda_{P V}\right)}+\frac{3 L v}{\pi} \log \Lambda_{P V} L} \lim _{\mu \rightarrow 0}\left(\mu^{4} e^{-I_{1} \log \mu^{2} L^{2}+\int_{\mu^{2}}^{\Lambda_{P V}^{2}} \frac{I_{2}\left(M^{2}\right)}{M^{2}} d M^{2}}\right)^{\frac{3}{4}} \\
=e^{-\frac{v L}{\pi} \frac{4 \pi^{2}}{g^{2}(1 / L)}} \lim _{\mu \rightarrow 0}\left(\mu^{4} e^{-I_{1} \log \mu^{2} L^{2}+\int_{\mu^{2}}^{\Lambda_{P}^{2}} \frac{I_{2}\left(M^{2}\right)}{M^{2}} d M^{2}}\right)^{\frac{3}{4}} \equiv e^{-\frac{v L}{\pi} \frac{4 \pi^{2}}{g^{2}(1 / L)}} R_{2},
\end{gathered}
$$

where $R_{2}$ is implicitly defined in the last line above. In other words, the UV divergent contribution to $R$ serves the purpose to renormalize the coupling from $\Lambda_{P V}$ to the scale $\frac{1}{L}$. Any other UV divergence at one loop would be in need of a counterterm and there is not another one at one loop.

Next, we consider $\log R_{2}$ (omitting the explicit mention of the $\mu \rightarrow 0$ limit to be taken at the end and noticing that the upper limit of the integral can be taken to infinity):

$$
\log R_{2}=\log \mu^{3}-\frac{3 I_{1}}{2} \log \mu L+\frac{3}{2} \int_{\mu}^{\infty} \frac{d M}{M}\left(\sum_{p} \frac{\frac{2 \pi p}{L}+v}{\left|\left(\frac{2 \pi p}{L}+v\right)^{2}+M^{2}\right|^{\frac{1}{2}}}-(v \rightarrow-v)\right) .
$$

Next, noting that $\int_{\mu}^{\infty} \frac{d M}{M \sqrt{A^{2}+M^{2}}}=\frac{1}{|A|} \operatorname{arcsinh} \frac{|A|}{\mu}=\frac{1}{|A|} \ln \frac{2|A|}{\mu}+\mathcal{O}\left(\mu^{2}\right)$, we find:

$$
\begin{aligned}
\log R_{2}=\log \mu^{3}-\frac{3 I_{1}}{2} \log \mu L & +\frac{3}{2} \sum_{p}\left(\operatorname{sign}\left(\frac{2 \pi p}{L}+v\right) \log \frac{2\left|\frac{2 \pi p}{L}+v\right|}{\mu}-(v \rightarrow-v)\right) \\
=\log \mu^{3}-\frac{3 I_{1}}{2} \log \mu L & +\frac{3}{2}\left[\sum_{p} \operatorname{sign}\left(p+\frac{v L}{2 \pi}\right)-(v \rightarrow-v)\right] \log \frac{4 \pi}{\mu L} \\
& +\frac{3}{2}\left[\sum_{p} \operatorname{sign}\left(p+\frac{v L}{2 \pi}\right) \log \left|p+\frac{v L}{2 \pi}\right|-(v \rightarrow-v)\right]
\end{aligned}
$$

Before continuing, we recognize from (A.7) that one of the KK sums appearing in (A.10), $\sum_{p} \operatorname{sign}\left(p+\frac{v L}{2 \pi}\right)-(v \rightarrow-v)=2-I_{1}$, giving rise, after substitution in (A.9), and remembering $I_{1}=\frac{2 L v}{\pi}$ :

$\log R_{2}$

$$
\begin{aligned}
& =\quad \log \mu^{3}-\frac{3 I_{1}}{2} \log \mu L+\frac{3}{2}\left(2-I_{1}\right) \log \frac{4 \pi}{\mu L}+\frac{3}{2}\left[\sum_{p} \operatorname{sign}\left(p+\frac{v L}{2 \pi}\right) \log \left|p+\frac{v L}{2 \pi}\right|-(v \rightarrow-v)\right] \\
& =\quad 3 \log \frac{4 \pi}{L}-3 \frac{L v}{\pi} \log 4 \pi+\frac{3}{2}\left[\sum_{p} \operatorname{sign}\left(p+\frac{v L}{2 \pi}\right) \log \left|p+\frac{v L}{2 \pi}\right|-(v \rightarrow-v)\right] .
\end{aligned}
$$


Now we can deal with the remaining KK sum, by writing it as an $s$-derivative of a function, evaluated at $s=0$ :

$$
\begin{aligned}
& \log R_{2} \\
& =3 \log \frac{4 \pi}{L}-3 \frac{L v}{\pi} \log 4 \pi-\left.\frac{3}{2} \frac{d}{d s}\left[\sum_{p} \frac{\operatorname{sign}\left(p+\frac{v L}{2 \pi}\right)}{\left|p+\frac{v L}{2 \pi}\right|^{s}}-(v \rightarrow-v)\right]\right|_{s \rightarrow 0} .
\end{aligned}
$$

Next, we define the function:

$$
H(s, a)=\sum_{p} \frac{\operatorname{sign}(p+a)}{|p+a|^{s}}
$$

and rewrite (A.12) as:

$$
\log R_{2}=3 \log \frac{4 \pi}{L}-3 \frac{L v}{\pi} \log 4 \pi-\frac{3}{2} \lim _{s \rightarrow 0} \frac{d}{d s}\left(H\left(s, \frac{v L}{2 \pi}\right)-H\left(s,-\frac{v L}{2 \pi}\right)\right) .
$$

Now, for $1>a>0$ we have:

$$
H(s, a)=\sum_{p \geq 0} \frac{1}{|p+a|^{s}}-\sum_{p \geq 0} \frac{1}{|p+1-a|^{s}}=\zeta(s, a)-\zeta(s, 1-a)
$$

where $\zeta(s, a)=\sum_{p \geq 0}|p+a|^{-s}$ is the incomplete zeta function, and similar for:

$$
H(s,-a)=\sum_{p \geq 0} \frac{1}{|p+1-a|^{s}}-\sum_{p \geq 0} \frac{1}{|p+a|^{s}}=\zeta(s, 1-a)-\zeta(s, a) .
$$

Thus, $R_{2}$ is given by:

$$
\log R_{2}=3 \log \frac{4 \pi}{L}-3 \frac{L v}{\pi} \log 4 \pi+3\left(\zeta^{\prime}\left(0,1-\frac{L v}{2 \pi}\right)-\zeta^{\prime}\left(0, \frac{L v}{2 \pi}\right)\right)
$$

which, upon plugging into (A.8), and using $\zeta^{\prime}(0, x)=\ln \Gamma(x)-\frac{1}{2} \log 2 \pi$ yields for $e^{-S_{0}}$ times the ratio of determinants $R$ in the BPS monopole background:

$$
\begin{aligned}
\left.e^{-S_{0}} R\right|_{B P S} & =\left(\frac{4 \pi}{L}\right)^{3} e^{-\frac{v L}{\pi}\left[\frac{4 \pi^{2}}{g^{2}(1 / L)}+3 \log 4 \pi\right]+3\left(\zeta^{\prime}\left(0,1-\frac{L v}{2 \pi}\right)-\zeta^{\prime}\left(0, \frac{L v}{2 \pi}\right)\right)} \\
& =\left(\frac{4 \pi}{L}\right)^{3} e^{-\frac{v L}{\pi} \frac{4 \pi^{2}}{g^{2}\left(\frac{4 \pi}{L}\right)}+3 \log \Gamma\left(1-\frac{L v}{2 \pi}\right)-3 \log \Gamma\left(\frac{v L}{2 \pi}\right)} .
\end{aligned}
$$

For the ratio of determinants around the KK monopole, we replace $\frac{v L}{2 \pi} \rightarrow 1-\frac{v L}{2 \pi}$, yielding:

$$
\left.e^{-S_{0}} R\right|_{K K}=\left(\frac{4 \pi}{L}\right)^{3} e^{-\frac{8 \pi^{2}}{g^{2}\left(\frac{4 \pi}{L}\right)}+\frac{v L}{\pi} \frac{4 \pi^{2}}{g^{2}\left(\frac{4 \pi}{L}\right)}-3 \log \Gamma\left(1-\frac{L v}{2 \pi}\right)+3 \log \Gamma\left(\frac{v L}{2 \pi}\right)} .
$$


We can now study the expansion of the $\Gamma$ functions from (A.18) and (A.19) near the center symmetric points:

$$
\begin{aligned}
3 \log \frac{\Gamma\left(1-\frac{L v}{2 \pi}\right)}{\Gamma\left(\frac{L v}{2 \pi}\right)} & =-3 \psi(1 / 2)\left(\frac{L v}{\pi}-1\right)+\mathcal{O}\left(\left(\frac{L v}{\pi}-1\right)^{3}\right) \\
& \approx 5.8903\left(\frac{L v}{\pi}-1\right)
\end{aligned}
$$

where we used the value $\psi(1 / 2)=\Gamma^{\prime}(1 / 2) / \Gamma(1 / 2) \approx-1.96$ of the digamma function. Thus, we can rewrite (A.18) as:

$$
\left.e^{-S_{0}} R\right|_{B P S}=\left(\frac{4 \pi}{L}\right)^{3} e^{-\frac{4 \pi^{2}}{g^{2}\left(\frac{4 \pi}{L}\right)}-\left(\frac{v L}{\pi}-1\right)\left(\frac{4 \pi^{2}}{g^{2}\left(\frac{4 \pi}{L}\right)}+3(\log \Gamma(1 / 2))^{\prime}\right)+\mathcal{O}\left(\left(\frac{L v}{\pi}-1\right)^{3}\right)},
$$

as well as a similar expression for the KK monopole (A.19). Thus, near the center symmetric point, we have that there is a small shift of the scale of the coupling constant away from $4 \pi / L$ (using $-1.96 \approx-\log 7.12$, to a scale a few times lower than $1 / R=2 \pi / L$ ) and that the exponential in $e^{-S_{0}} R$ is a linear function of the deviation from the center symmetric vacuum, $\frac{v L}{\pi}-1$, up to cubic terms. The behavior of the ratio of one-loop determinants is illustrated for general $v L$ in Figure 2.

It is of interest to also consider the neighborhood of $v L=0$, in particular, if we wish to take the 3d limit and compare with previous studies as a check on our calculation. In the 3 d limit, we take $L \rightarrow 0$, but keep $g_{3}^{2}=L / g_{4}^{2}$ fixed; as before, $v$ is an arbitrary position on the moduli space, which becomes noncompact in the $3 \mathrm{~d}$ limit. The contribution of the KK monopole vanishes in the $3 \mathrm{~d}$ limit, as its action becomes infinite. Hence, we concentrate on the BPS monopole amplitude. In this limit, we have that:

$$
\left.3 \log \frac{\Gamma\left(1-\frac{L v}{2 \pi}\right)}{\Gamma\left(\frac{L v}{2 \pi}\right)}\right|_{L v \approx 0}=-3 \log \frac{2 \pi}{L v}+3 \gamma_{E} \frac{L v}{\pi}+\mathcal{O}\left(\left(\frac{L v}{\pi}\right)^{3}\right),
$$

so we can write for (A.18):

$$
\begin{aligned}
\left.e^{-S_{0}} R\right|_{B P S} & =\left(\frac{4 \pi}{L}\right)^{3} e^{-\frac{v L}{\pi} \frac{4 \pi^{2}}{g^{2}\left(\frac{4 \pi}{L}\right)}-3 \log \frac{2 \pi}{v L}+3 \gamma_{E} \frac{L v}{\pi}+\mathcal{O}\left(\left(\frac{L v}{\pi}\right)^{3}\right)} \\
& \rightarrow(2 v)^{3} e^{-\frac{4 \pi v}{g_{3}^{2}}}, \text { with } L \rightarrow 0, \text { fixed } g_{3}^{2}=\frac{L}{g_{4}^{2}\left(\frac{4 \pi}{L}\right)}
\end{aligned}
$$

We note that in this limit, our calculation exactly reproduces the $3 \mathrm{~d}$ result of [41]. To facilitate the comparison, we need to take into account the facts that $v=m_{W}$ in our normalization and that our calculation is in the 4-supercharges theory, not the 8-supercharges one, which changes the overall power of the dimensional pre factor from 2 , as in eqn. (26) from [41], to 3 as in our (A.23). It is clear from the above that the noncancellation of the determinants is required in order to have a smooth $3 \mathrm{~d}$ limit. 


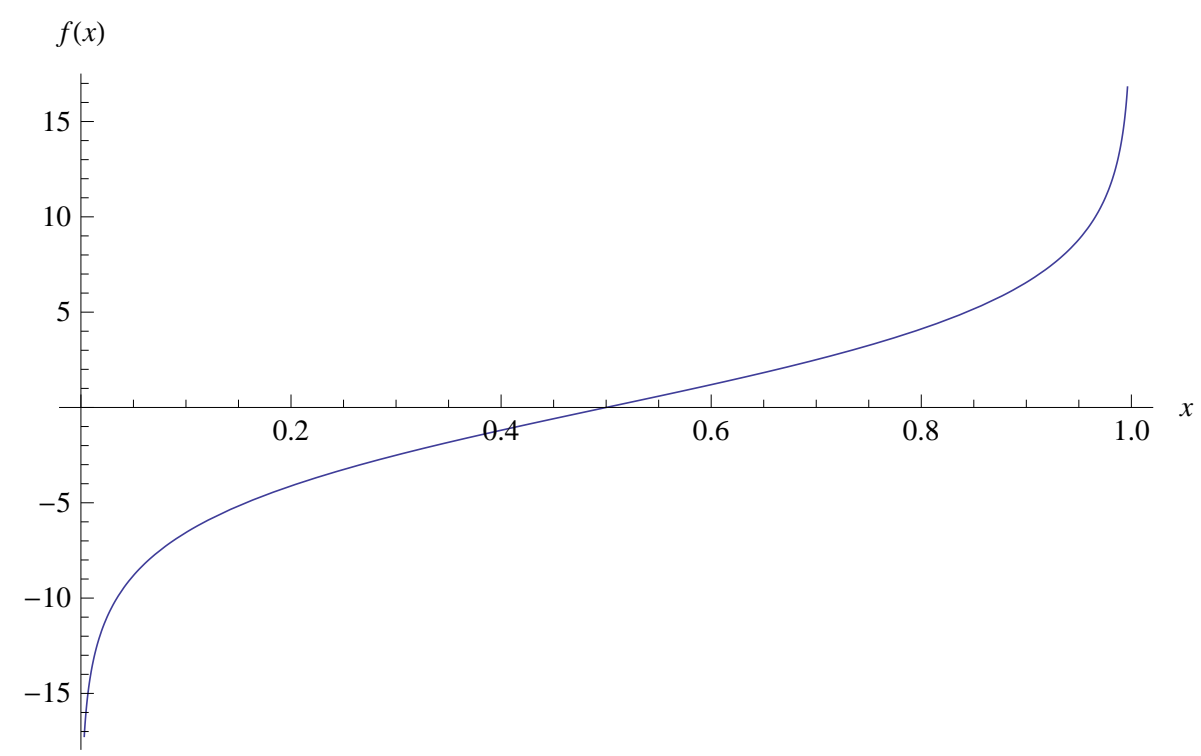

Figure 2: The behavior of the ratio of nonzero mode determinants around a BPS monopole-instanton, eqn. (A.18). We define $f(x)=3(\log \Gamma(1-x)-\log \Gamma(x))$, where $x=v L / 2 \pi$. The singularities of $f(x)$ near the gauge-symmetry restoration points $(x=0,1)$ are logarithmic and are, in fact, required in order to take the proper $3 \mathrm{~d}$ limit, see discussion around eqn. (A.23)). Notice that at the centersymmetric point the nonzero modes determinants cancel exactly, $f(1 / 2)=0$, while the slope there is $-3 \psi(1 / 2) \simeq 5.89$, see eqn. (A.20).

\section{A.2 Interpretation}

The main question is, what is the interpretation of the cubic and higher terms in $f(x)$ away from the center symmetric point? It appears at first sight that they do not belong in the superpotential, as they are not holomorphic functions of $v L+i \sigma$. Further, any nonlinear terms in this variable appearing in the exponent would be in conflict with symmetry arguments.

We note that these questions arise already in the $3 \mathrm{~d}$ supersymmetric theories and have been addressed in some cases. Notably, in the 8-supercharges $3 \mathrm{~d}$ theory, the BPS monopoleinstanton induced terms give rise to the four-fermi interaction [41]:

$$
\frac{v}{\left(g_{3}^{2}\right)^{4}} \lambda^{2} \psi^{2} e^{-\frac{4 \pi v}{g_{3}^{2}}+i \sigma}
$$

Since the 8-supercharge theory admits no superpotential, this term, including the $v$-dependent prefactor should be interpreted as arising from a component expansion of a supersymmetric sigma model with a hyper-Käbler metric. In fact, as shown in [41], the above four-fermi term is fully consistent with the semiclassical expansion of the Atiyah-Hitchin metric.

On the other hand, in the 4-supercharges 3d theory, we have, from (A.23), after incorporating bosonic and fermionic zero modes, the following form for the monopole-instanton 
induced fermion vertex:

$$
\frac{v^{3}}{\left(g_{3}^{2}\right)^{3}} \lambda^{2} e^{-\frac{4 \pi v}{g_{3}^{2}}+i \sigma} .
$$

It is natural to ask how this term is absorbed in an $e^{-X}$ superpotential, where $X=\frac{4 \pi v}{g_{3}^{2}}-i \sigma$, as argued long time ago [48]. The pre-exponential factor there and in subsequent work was assumed to be a modulus-independent constant and has not been calculated before, to the best of our knowledge. Thus the question regarding the incorporation of the nonholomorphic $\sim v^{3}$ prefactor in a supersymmetric effective lagrangian applies to the $3 \mathrm{~d} 4$-supercharge theory as well.

We now proceed to address this issue by first writing down the fermion vertices that accompany the BPS (A.18) and KK (A.19) monopole-instantons, performing the same steps as in [12]. We skip the details (of including the collective coordinates measure, etc., as they are identical to [12]) and note that the only difference is that we replace the Pauli-Villars scale appearing in the fermion measure there with the ratio of determinants that we calculated above. The fermion bilinear terms - the kinetic term and the ones that arise due to the BPS and KK monopole-instantons - are (all couplings below are taken at the scale $\frac{4 \pi}{L}$ ):

$$
\begin{aligned}
L_{f e r m}= & \frac{L}{g^{2}} i \bar{\lambda} \sigma^{m} \partial_{m} \lambda \\
& +\left(\frac{L}{g^{2}}\right)^{2} \frac{2^{4} \pi^{2} L}{g^{2}}\left(\frac{4 \pi}{L}\right)^{3}\left[e^{-\frac{v L}{\pi} \frac{4 \pi^{2}}{g^{2}}+i \sigma+f\left(\frac{v L}{2 \pi}\right)}+e^{-\frac{8 \pi^{2}}{g^{2}}+\frac{v L}{\pi} \frac{4 \pi^{2}}{g^{2}}-i \sigma-f\left(\frac{v L}{2 \pi}\right)}\right] \lambda \lambda,
\end{aligned}
$$

where

$$
e^{f(x)}=\left(\frac{\Gamma(1-x)}{\Gamma(x)}\right)^{3}
$$

was already defined in the caption of Figure 2. For future interpretation, it is also useful to recall the original, not resummed, expression for $f(x)$. This follows from (A.11) and, as given below, is valid for $0<x<1$ :

$$
f(x)=3 \ln x+3 \sum_{p>0} \ln (p+x)-\ln (p-x) .
$$

Next, we rewrite (A.24), using $b=\frac{4 \pi}{g^{2}} v L$ and expanding around the center-symmetric value $b \equiv \frac{4 \pi^{2}}{g^{2}}+b^{\prime}$ (in other words, $b^{\prime}$ is the fluctuation around the center symmetric vev):

$$
\begin{aligned}
L_{f e r m}= & \frac{L}{g^{2}} i \bar{\lambda} \sigma^{m} \partial_{m} \lambda+\frac{1}{2} \frac{g^{2}}{16 \pi^{2} L} \partial_{m} b^{\prime} \partial^{m} b^{\prime}+\ldots \\
& +\frac{2^{10} \pi^{5}}{g^{6}} e^{-\frac{4 \pi^{2}}{g^{2}}}\left[e^{-b^{\prime}+i \sigma} e^{f\left(\frac{1}{2}+\frac{g^{2}}{8 \pi^{2}} b^{\prime}\right)}+e^{b^{\prime}-i \sigma} e^{-f\left(\frac{1}{2}+\frac{g^{2}}{8 \pi^{2}} b^{\prime}\right)}\right] \lambda \lambda,
\end{aligned}
$$

where we included the kinetic term for $b^{\prime}$ to leading order (subleading contributions to the $b^{\prime}$ kinetic term, to be elaborated below, are denoted by dots). The main question we want to address is how (A.27) can be incorporated in a supersymmetric effective lagrangian, given 
the extra, seemingly nonholomorphic, dependence of the fermion 't Hooft vertex on $b^{\prime}-i \sigma$, arising from the noncanceling determinants around the BPS and KK monopole-instantons.

For the reader not so interested in the details, the summary of the discussion of the following section is that after properly performing the supersymmetric "photon-dual photon" duality transformation, the dimensionless chiral superfield $B$ dual to the abelian vector multiplet (in $3 \mathrm{~d}$, equivalent to a real linear multiplet), describing $A_{4}$ and the $3 \mathrm{~d}$ photon, is such that its lowest component is:

$$
B \mid=b^{\prime}-f\left(\frac{1}{2}+\frac{g^{2}}{8 \pi^{2}} b^{\prime}\right)-i \sigma .
$$

In terms of the chiral superfield (A.28), the superpotential is holomorphic and is of the wellknown affine-Toda form, $W \sim e^{-B}+e^{B}$. In the next Section, we discuss the details of the duality transformation and the interpretation of the function $f$ in (A.28) as encoding perturbative corrections to the moduli space metric on $\mathbb{R}^{3} \times \mathbb{S}^{1}$. We recall again that $f$ is nonzero only away from the center-symmetric point, thus the extremal point of $W(B)$, $B=0$, still corresponds to the center-symmetry preserving vacuum $b^{\prime}=0$, in accord with the conclusions from the earlier work $[12,40]$.

\section{A.3 Linear-chiral superfield duality}

The dimensional reduction of an abelian $4 \mathrm{~d}$ vector multiplet to $3 \mathrm{~d}$ is described as a real linear multiplet, $W$, defined as: ${ }^{11}$

$$
W=\frac{1}{2} \bar{\sigma}^{3 \dot{\alpha} \alpha} \bar{D}_{\dot{\alpha}} D_{\alpha} V,
$$

where $V$ is the usual real vector superfield in 4 d (dimensional reduction in (A.29) as written is performed along the $x^{3}$ direction and, hence, the lowest component of (A.29) is $W \mid=A_{3}$ ). The field $W$ obeys the linear multiplet relations $D^{2} W=\bar{D}^{2} W=0$ and is invariant under the usual (3d) supergauge transformations $V \rightarrow V-i\left(\Lambda-\Lambda^{\dagger}\right)$. The lowest component of $W$, as already indicated, is the real scalar in the $3 \mathrm{~d}$ vector mulitiplet (i.e., is related by rescaling to our fields $b$ or $b^{\prime}$ ), while the other terms in its superspace expansion involve the fermions and the $U(1)$ gauge field strength. As defined above, the dimension of $W$ is unity. The minimal kinetic term for the vector multiplet is given by the first term in the $D$-term action given below:

$$
\int d^{4} \theta d^{3} x\left(-\frac{1}{2 e^{2}} W^{2}-F(W)\right)
$$

while the function $F(W)$ incorporates possible nonlinear corrections to the kinetic terms. The action (A.30) is the most general two-derivative one involving the linear multiplet (note that $W$ can not appear in integrals over half-superspace). The coupling $e^{2}$ in (A.30) has unit mass dimension and denotes the $3 \mathrm{~d}$ gauge coupling; clearly, $e^{2}$ or another mass scale must be present in $F(W)$. The kinetic term of the lowest component of $W$, which we denote by $w$ in this Section $(W \mid \equiv w)$, hoping not to cause confusion, reads:

$$
\int d^{3} x \frac{1}{2}\left(\frac{1}{e^{2}}+F^{\prime \prime}(w)\right) \partial_{m} w \partial^{m} w
$$

\footnotetext{
${ }^{11}$ Our notation, including the supercovariant derivatives and $V$, is that of Wess and Bagger [47].
} 
For the theory to make sense, the function $\frac{1}{e^{2}}+F^{\prime \prime}(w)$ (the "tau-parameter," or moduli space metric), which also determines the effective $U(1)$ coupling along the Coulomb branch, has to be positive.

Linear-chiral duality is performed via a Legendre transformation as follows:

$$
\int d^{4} \theta d^{3} x\left(-\frac{1}{2 e^{2}} W^{2}-F(W)+\left(B^{\dagger}+B\right) W\right) .
$$

The dimensionless chiral superfield $B$ is introduced as a Lagrange multiplier and $W$ is now regarded as an unconstrained real superfield. Variation with respect to $B$ and $B^{\dagger}$, taking into account the chirality constraint $D_{\alpha} B^{\dagger}=\bar{D}_{\dot{\alpha}} B=0$, enforces the linear multiplet conditions $\bar{D}^{2} W=D^{2} W=0$ on the real superfield $W$.

On the other hand, a variation of (A.32) with respect to $W$ gives: ${ }^{12}$

$$
B+B^{\dagger}=\frac{1}{e^{2}} W+F^{\prime}(W) .
$$

The equation of motion (A.33) can be inverted, e.g. perturbatively, to give $W=e^{2}(B+$ $\left.B^{\dagger}\right)+\ldots$, and upon plugging the solution of (A.33) in (A.32), one is left with a dual theory of chiral superfields given by:

$$
\int d^{4} \theta d^{3} x K\left(B+B^{\dagger}\right)=\int d^{4} \theta d^{3} x\left(\frac{e^{2}}{2}\left(B+B^{\dagger}\right)^{2}+\ldots\right)=\int d^{4} \theta d^{3} x\left(e^{2} B^{\dagger} B+\ldots\right),
$$

where the dots denote higher order terms determined by the form of $F(W)$. For a recent reference, see, for example, [49]. The point of the discussion above is that the relation between the real part of the lowest component of the dual chiral superfield $B$ and the lowest component of the linear superfield $W$ is simple-i.e., linear-only if the function $F(W)$ is quadratic, i.e., if there are no nontrivial corrections to the kinetic terms of the $b^{\prime} \sim w$ field. In general, this relation can be complicated.

In particular, in our case of interest, the duality transformation on $\mathbb{R}^{3} \times \mathbb{S}^{1}$ is performed after Kaluza-Klein modes are integrated out. Integrating out the KK modes generates nontrivial corrections to the kinetic terms of $A_{4}$ and $3 \mathrm{~d}$ photon. As we will now see, these perturbative corrections are precisely related to the function $f$ (A.26) representing the ratio of nonzero modes' fermionic and bosonic determinants around the BPS and KK monopole instantons. To see this, let us now apply the duality transformation to our theory. As already noted, eqn. (A.33) determines the lowest component of $X$ in terms of $w(x)$ and the dual photon $\sigma(x)$ :

$$
B \mid=\frac{1}{2 e^{2}} w+\frac{1}{2} F^{\prime}(w)-i \sigma,
$$

which, upon comparison to (A.28) immediately implies that:

$$
b^{\prime}(x) \equiv \frac{1}{2 e^{2}} w(x) \text { and } F^{\prime}(w) \equiv-2 f\left(\frac{1}{2}+\frac{g^{2}}{16 \pi^{2} e^{2}} w\right) .
$$

\footnotetext{
${ }^{12}$ Notice that this relation also implies that the fermion components of $B$ are slightly different from the fermionic components of $W$; we will ignore this in what follows.
} 
Then, we can use (A.36) to compare the leading order kinetic term of $w$, eqn. (A.31), with that of $b^{\prime}$, eqn. (A.27), and find:

$$
e^{2}=\frac{g^{2}}{64 \pi^{2} L}
$$

We can now use the explicit form (A.26) of $f$ and (A.37) to find that the corrections to the kinetic term for $w$ (A.31) are determined by the effective "tau parameter":

$$
\begin{aligned}
\frac{1}{e^{2}}+F^{\prime \prime}(w) & =\frac{64 \pi^{2} L}{g^{2}}-8 L f^{\prime}\left(\frac{1}{2}+4 L w\right) \\
& =\frac{64 \pi^{2} L}{g^{2}}-\left.24 L\left[\frac{1}{x}+\sum_{p>0} \frac{1}{p-x}+\frac{1}{p+x}\right]\right|_{x \rightarrow \frac{1}{2}+4 L w} .
\end{aligned}
$$

In the last line, we used eqn. (A.26) for $f(x)$ and denoted $f^{\prime}(x) \equiv d f(x) / d x$.

In order to interpret the corrections, we rewrite (A.38) as:

$$
\frac{1}{e^{2}}+F^{\prime \prime}(w)=64 \pi^{2}\left(\frac{L}{g^{2}}-\left.\frac{3 L}{8 \pi^{2}}\left[\frac{1}{x}+\sum_{p>0} \frac{1}{p-x}+\frac{1}{p+x}\right]\right|_{x \rightarrow \frac{1}{2}+4 L w}\right)
$$

It is most straightforward to first consider the purely-3d limit $L \rightarrow 0, \frac{L}{g^{2}}$-fixed. In this case, the sum over $p>0$ in (A.40) drops out and only the first term in the square brackets survives. Recall that $g^{2}$ is taken at the scale $\frac{4 \pi}{L}$, which is now the $3 \mathrm{~d}$ UV cutoff scale (the bare $3 \mathrm{~d}$ coupling is $\frac{L}{g^{2}}$ ). The coupling (A.40) can be rewritten, using $x=\frac{v L}{2 \pi}$, as:

$$
\frac{1}{e^{2}}+F^{\prime \prime}(w)=64 \pi^{2}\left(\frac{L}{g^{2}}-\frac{3 L}{8 \pi^{2}} \frac{2 \pi}{v L}\right)=64 \pi^{2}\left(\frac{L}{g^{2}}-\frac{3}{4 \pi v}\right) .
$$

The $\frac{3}{4 \pi v}$ shift of $\frac{L}{g^{2}}$ in (A.41) represents exactly the one-loop shift of the bare $3 \mathrm{~d}$ gauge coupling $\frac{L}{g^{2}}$ due to integrating out the 3 d heavy vector multiplet - the heavy $W$-boson and superpartner, of mass $\sim v$, along the Coulomb branch parameterized by $v$ in an $S U(2)$ theory. This one-loop shift was calculated in [50], see eqn. (83) there. ${ }^{13}$ Note that we obtained the same result as [50] by a rather roundabout way - by calculating the ratio of determinants of nonzero modes in the monopole-instanton background and then demanding consistency of the result with holomorphy of the superpotential. While this may appear miraculous, the matching of the results had to be true, by the power of supersymmetry.

Going back to finite- $L$, i.e. to $\mathbb{R}^{3} \times \mathbb{S}^{1}$, the interpretation of the other terms appearing in (A.40) is now clear. The sum over $p>0$ represents the effect of the Kaluza-Klein partners of the heavy vector multiplets on the unbroken- $U(1)$ gauge coupling; recall that at every KK level with $p>0$, there are two heavy vector multiplets of mass $\frac{2 p \pi}{L} \pm v$. We are not aware of an explicit perturbative calculation of this in a compactified $N=1$ theory however, similar to

\footnotetext{
${ }^{13}$ The factor of $64 \pi^{2}$ represents an overall normalization factor; note that ref. [50] calculated precisely the "running" of the $3 \mathrm{~d}$ coupling from the bare value $\frac{L}{g^{2}}$ to the value at scales below $v$, with a result exactly as given in the brackets in (A.41).
} 
the purely-3d four supercharge case and to the $N=24 \mathrm{~d}$ theory, ${ }^{14}$ and given the suggestive form of the KK sum in (A.40), it is natural to conjecture that, by supersymmetry, the nonvanishing ratio of nonzero modes determinants around the monopole-instanton solutions precisely encodes the one-loop corrections to the $U(1)$ gauge coupling.

In our discussion in the main text, we have ignored the non-canceling nonzero mode determinants. This is because their net effect is to modify the Kähler potential for the chiral superfield $B$, see eqn. (A.35), by small loop-suppressed terms. Our main concern is the competition between nonperturbative effects at weak coupling, while the perturbative deformation of the Kähler potential (2.10) provides only small corrections to the leading order semiclassical results.

\section{B. The monopole-induced potential in softly-broken SYM}

In this appendix, we determine the prefactor of the monopole instanton amplitude in softly broken $\mathcal{N}=1$ theory on $\mathbb{R}^{3} \times \mathbb{S}^{1}$. From [12], the collective coordinate measure for $\mathcal{M}_{1}$ (BPS) monopole-instantons in $S U(2)$ gauge theory, is:

$$
\int d \mu_{B P S}=\frac{M_{P V}^{3} L}{2 \pi g^{2}} \int d^{3} a d \phi d^{2} \zeta e^{-b+i \sigma},
$$

where $b=\langle b\rangle+b(a), \sigma=\langle\sigma\rangle+\sigma(a)$. The three-dimensional vector $a$ denotes the collective coordinate representing the center of the monopole, $\phi$ is the angular $U(1)$ collective coordinate, $\zeta^{\alpha}$ are the two Grassmann fermion zero mode coordinates, and the prefactor represents the product of all collective coordinate Jacobians.

Now, we add the fermion mass term $\Delta L_{m}=\frac{m}{g^{2}} \operatorname{tr}[\lambda \lambda]$, see (2.32), to the Lagrangian of SYM. In order to obtain the contribution of, for example, the $\mathcal{M}_{1}$ (or BPS) monopoleinstanton to the potential for $b$ and $\sigma$, we saturate the integral over the fermion zero modes by a single mass-term insertion:

$$
\int d \mu_{B P S} e^{-\Delta S_{m}} \approx-\frac{M_{P V}^{3} L}{g^{2}} \int d^{3} a e^{-b+i \sigma} \frac{m L}{g^{2}} \int d^{2} \zeta d^{3} x \operatorname{tr}\left[\lambda^{0}(x-a) \lambda^{0}(x-a)\right],
$$

where $\Delta S_{m}=\int d^{4} x \Delta L_{m}$ and $\lambda^{0}(x-a)$ is the fermion zero mode of the monopole located at $a$. Next we note that $\frac{L}{g^{2}} \int d^{2} \zeta d^{3} x \operatorname{tr}\left[\lambda^{0} \lambda^{0}\right]$ is exactly the fermion zero-mode Jacobian calculated in [41], which is equal to $2 S_{c l}^{\mathcal{M}_{1}}=2 b$. Collecting all factors, we conclude that the contribution of a $\mathcal{M}_{1}$ monopole-instanton to the potential is:

$$
\Delta V^{\mathcal{M}_{1}}=\frac{2 m M_{P V}^{3} L}{g^{2}} b e^{-b+i \sigma} .
$$

Proceeding similarly, we find that the $\mathcal{M}_{2}$ (or KK) monopole-instanton contributes, using its action $S_{c l}^{\mathcal{M}_{2}}=\frac{8 \pi^{2}}{g^{2}}-b$ instead:

$$
\Delta V^{\mathcal{M}_{2}}=\frac{2 m M_{P V}^{3} L}{g^{2}}\left(\frac{8 \pi^{2}}{g^{2}}-b\right) e^{-\frac{8 \pi^{2}}{g^{2}}+b-i \sigma}
$$

\footnotetext{
${ }^{14}$ See Section 4.1 in [51] for an expression, similar to (A.40), for the one-loop correction to the moduli space metric of the $N=2$ theory on $\mathbb{R}^{3} \times \mathbb{S}^{1}$, valid also on the Coulomb branch with only a Wilson line turned on.
} 
To obtain the total $\mathcal{O}(m)$ contribution to the scalar potential, we now sum over the contributions of the two monopole-instantons and their complex conjugates:

$$
\begin{aligned}
V_{\text {mon. }} & =\Delta V^{\mathcal{M}_{1}}+\Delta V^{\mathcal{M}_{2}}+\text { h.c. } \\
& =\frac{32 \pi^{2} m M_{P V}^{3} L}{g^{4}} e^{-\frac{4 \pi^{2}}{g^{2}}} \cos \sigma\left(\cosh b^{\prime}-\frac{g^{2}}{4 \pi^{2}} b^{\prime} \sinh b^{\prime}\right) .
\end{aligned}
$$

Finally, we use the relations (2.16), to cast $V_{\text {mon. }}$ into the form given in (2.33).

\section{References}

[1] D. J. Gross, R. D. Pisarski and L. G. Yaffe, "QCD and instantons at finite temperature," Rev. Mod. Phys. 53, 43 (1981).

[2] V. M. Belyaev, "Higher Loop Contributions To Effective Potential Of Gauge Theory At High Temperature," Phys. Lett. B 241, 91 (1990).

[3] K. Enqvist and K. Kajantie, "Hot Gluon Matter In A Constant A(0) Background," Z. Phys. C 47, 291 (1990).

[4] C. P. Korthals Altes, "Constrained effective potential in hot QCD," Nucl. Phys. B 420, 637 (1994) [hep-th/9310195].

[5] M. Ünsal and L. G. Yaffe, "Large-N volume independence in conformal and confining gauge theories," JHEP 1008, 030 (2010) [arXiv:1006.2101 [hep-th]].

[6] T. Schäfer and F. Wilczek, "Continuity of quark and hadron matter," Phys. Rev. Lett. 82, 3956 (1999) [hep-ph/9811473].

[7] P. N. Meisinger and M. C. Ogilvie, "Complete high temperature expansions for one loop finite temperature effects," Phys. Rev. D 65, 056013 (2002) [hep-ph/0108026].

[8] K. -M. Lee, P. Yi, "Monopoles and instantons on partially compactified D-branes," Phys. Rev. D56, 3711-3717 (1997). [hep-th/9702107].

[9] T. C. Kraan and P. van Baal, "Monopole constituents inside $S U(n)$ calorons," Phys. Lett. B 435, 389 (1998) [arXiv:hep-th/9806034].

[10] T. M. W. Nye and M. A. Singer, "An $\mathcal{L}^{2}$-index theorem for Dirac operators on $\mathbb{R}^{3} \times \mathbb{S}^{1}$," J. Funct. Anal. 177, 203 (2000); arXiv:math/0009144.

[11] E. Poppitz and M. Ünsal, "Index theorem for topological excitations on $\mathbb{R}^{3} \times \mathbb{S}^{1}$ and Chern-Simons theory," JHEP 0903, 027 (2009) [arXiv:0812.2085 [hep-th]].

[12] N. M. Davies, T. J. Hollowood and V. V. Khoze, "Monopoles, affine algebras and the gluino condensate," J. Math. Phys. 44, 3640 (2003) [hep-th/0006011].

[13] M. Ünsal, "Abelian duality, confinement, and chiral symmetry breaking in QCD(adj)," Phys. Rev. Lett. 100, 032005 (2008) [arXiv:0708.1772 [hep-th]].

[14] M. Ünsal, "Magnetic bion condensation: A new mechanism of confinement and mass gap in four dimensions," Phys. Rev. D 80 (2009) 065001 [arXiv:0709.3269 [hep-th]].

[15] M. M. Anber, E. Poppitz, "Microscopic structure of magnetic bions," JHEP 1106, 136 (2011). [arXiv:1105.0940 [hep-th]]. 
[16] E. B. Bogomolny, "Calculation of instanton-anti-instanton contributions in quantum mechanics," Phys. Lett. B91, 431-435 (1980).

[17] J. Zinn-Justin, "Multi-instanton contributions in quantum mechanics," Nucl. Phys. B192, 125-140 (1981).

[18] T. Schäfer and E. V. Shuryak, "Instantons in QCD," Rev. Mod. Phys. 70, 323 (1998) [hep-ph/9610451].

[19] P. Argyres and M. Ünsal, "A semiclassical realization of infrared renormalons," arXiv:1204.1661 [hep-th].

[20] D. Diakonov, "How to check that dyons are at work?," arXiv:1012.2296 [hep-ph].

[21] I. I. Balitsky, A. V. Yung, "Instanton molecular vacuum in $N=1$ supersymmetric quantum mechanics," Nucl. Phys. B274, 475 (1986).

[22] F. Karsch and E. Laermann, "Thermodynamics and in medium hadron properties from lattice QCD," In *Hwa, R.C. (ed.) et al.: Quark gluon plasma* 1-59 [hep-lat/0305025].

[23] E. Poppitz, M. Ünsal, "Seiberg-Witten and 'Polyakov-like' magnetic bion confinements are continuously connected," JHEP 1107, 082 (2011). [arXiv:1105.3969 [hep-th]].

[24] D. Diakonov and V. Petrov, "Confining ensemble of dyons," Phys. Rev. D 76, 056001 (2007) [arXiv:0704.3181 [hep-th]].

[25] G. 't Hooft, "Topology of the Gauge Condition and New Confinement Phases in Nonabelian Gauge Theories," Nucl. Phys. B 190, 455 (1981).

[26] O. Aharony, J. Marsano, S. Minwalla, K. Papadodimas and M. Van Raamsdonk, "A First order deconfinement transition in large- $N$ Yang-Mills theory on a small $\mathbb{S}^{3}$," Phys. Rev. D 71, 125018 (2005) [hep-th/0502149].

[27] D. Simic and M. Ünsal, "Deconfinement in Yang-Mills theory through toroidal compactification with deformation," [arXiv:1010.5515 [hep-th]].

[28] M. M. Anber, E. Poppitz and M. Ünsal, "2d affine XY-spin model/4d gauge theory duality and deconfinement," JHEP 1204, 040 (2012) [arXiv:1112.6389 [hep-th]].

[29] D. Diakonov, "Instantons at work," Prog. Part. Nucl. Phys. 51, 173 (2003) [hep-ph/0212026].

[30] D. Diakonov, "Topology and confinement," Nucl. Phys. Proc. Suppl. 195, 5 (2009) [arXiv:0906.2456 [hep-ph]].

[31] A. M. Polyakov, "Quark Confinement and Topology of Gauge Groups," Nucl. Phys. B 120, 429 (1977).

[32] M. Ünsal and L. G. Yaffe, "Center-stabilized Yang-Mills theory: Confinement and large- $N$ volume independence," Phys. Rev. D 78, 065035 (2008) [arXiv:0803.0344 [hep-th]].

[33] A. Dumitru, Y. Hatta, J. Lenaghan, K. Orginos and R. D. Pisarski, "Deconfining phase transition as a matrix model of renormalized Polyakov loops," Phys. Rev. D 70, 034511 (2004) [hep-th/0311223].

[34] R. D. Pisarski, "Effective Theory of Wilson Lines and Deconfinement," Phys. Rev. D 74, 121703 (2006) [hep-ph/0608242].

[35] A. Dumitru, Y. Guo, Y. Hidaka, C. P. K. Altes and R. D. Pisarski, "How Wide is the Transition to Deconfinement?," Phys. Rev. D 83, 034022 (2011) [arXiv:1011.3820 [hep-ph]]. 
[36] A. Dumitru, Y. Guo, Y. Hidaka, C. P. K. Altes and R. D. Pisarski, "Effective matrix model for deconfinement in pure gauge theories," arXiv:1205.0137 [hep-ph].

[37] J. C. Myers and M. C. Ogilvie, "New phases of SU(3) and SU(4) at finite temperature," Phys. Rev. D 77, 125030 (2008) [arXiv:0707.1869 [hep-lat]].

[38] F. Bruckmann, T. G. Kovacs and S. Schierenberg, "Anderson localization through Polyakov loops: lattice evidence and Random matrix model," Phys. Rev. D 84, 034505 (2011) [arXiv:1105.5336 [hep-lat]].

[39] M. D'Elia and F. Negro, " $\theta$ dependence of the deconfinement temperature in Yang-Mills theories," Phys. Rev. Lett. 109, 072001 (2012) [arXiv:1205.0538 [hep-lat]].

[40] N. M. Davies, T. J. Hollowood, V. V. Khoze and M. P. Mattis, "Gluino condensate and magnetic monopoles in supersymmetric gluodynamics," Nucl. Phys. B 559, 123 (1999) [hep-th/9905015].

[41] N. Dorey, V. V. Khoze, M. P. Mattis, D. Tong and S. Vandoren, "Instantons, three-dimensional gauge theory, and the Atiyah-Hitchin manifold," Nucl. Phys. B 502, 59 (1997) [hep-th/9703228].

[42] E. J. Weinberg, "Parameter Counting for Multi-Monopole Solutions," Phys. Rev. D 20, 936 (1979).

[43] R. K. Kaul, "Monopole Mass In Supersymmetric Gauge Theories," Phys. Lett. B 143, 427 (1984).

[44] A. Rebhan, P. van Nieuwenhuizen, R. Wimmer, "Quantum corrections to solitons and BPS saturation," arXiv:0902.1904

[45] H. -Y. Chen, N. Dorey and K. Petunin, "Wall Crossing and Instantons in Compactified Gauge Theory," JHEP 1006, 024 (2010) [arXiv:1004.0703 [hep-th]].

[46] S. Vandoren and P. van Nieuwenhuizen, "Lectures on instantons," arXiv:0802.1862 [hep-th].

[47] J. Wess and J. Bagger, "Supersymmetry and supergravity", 2nd ed., Princeton U.P., 1992.

[48] I. Affleck, J. A. Harvey and E. Witten, "Instantons and (Super)Symmetry Breaking in (2+1)-Dimensions," Nucl. Phys. B 206, 413 (1982).

[49] J. de Boer, K. Hori and Y. Oz, "Dynamics of $\mathrm{N}=2$ supersymmetric gauge theories in three-dimensions," Nucl. Phys. B 500, 163 (1997) [hep-th/9703100].

[50] A. V. Smilga and A. Vainshtein, "Background field calculations and nonrenormalization theorems in 4-D supersymmetric gauge theories and their low-dimensional descendants," Nucl. Phys. B 704, 445 (2005) [hep-th/0405142].

[51] D. Gaiotto, G. W. Moore and A. Neitzke, "Four-dimensional wall-crossing via three-dimensional field theory," Commun. Math. Phys. 299, 163 (2010) [arXiv:0807.4723 [hep-th]]. 